\title{
Deuterium retention in solid and liquid tin after low-temperature plasma exposure
}

\author{
A. Manhard*, T. Schwarz-Selinger, M. Balden, T. Dürbeck, H. Maier, R. Neu \\ Max Planck Institute for Plasma Physics, Boltzmannstr. 2, 85748 Garching, Germany \\ *corresponding author: armin.manhard@ipp.mpg.de \\ Published as: A. Manhard et al, Nucl. Fusion 60 (2020) 106007 \\ https://doi.org/10.1088/1741-4326/aba801
}

\begin{abstract}
Recently, plasma-facing components based on liquid metals were proposed for a prospective nuclear fusion reactor in order to circumvent challenges that occur for solid metal components, such as erosion lifetime, neutron embrittlement or transient overloading. One candidate material is liquid tin. We present a systematic study of the behavior of solid and liquid tin exposed to a lowtemperature deuterium plasma at temperatures between 300 and $515 \mathrm{~K}$, focusing on deuterium retention and thermal release as well as on tin erosion and re-deposition. We find strong variations of deuterium depth profiles, release spectra, and erosion rates, which are correlated to dramatic changes in the surface morphology. In particular, we find evidence for massive gas bubble formation in tin, which can lead to the evolution of a thick, sponge-like layer for tin exposed to deuterium plasma just below the melting point, and to the rapid formation of a macroscopic gas pocket below liquid tin. We present evidence for strongly temperature-dependent chemical erosion of tin by deuterium plasma in the solid state. In the liquid state, a high tin erosion rate occurs, which is apparently induced by ejection of tin microdroplets. Furthermore, we observed strong plasmaassisted, low-temperature wetting of tin on tungsten near the melting point of tin. We tentatively propose the hypothesis that all of the observed effects may be influenced by tin-deuterium chemistry, e.g., the formation and de-composition of stannane molecules.
\end{abstract}

\section{Introduction}

In a future nuclear fusion reactor, the plasma-facing components (PFCs) will be subjected to intense heat and energetic particle fluxes, particularly in the divertor region. Only few materials have the potential to withstand this environment, while at the same time fulfilling the conditions that the uptake of the rare and radioactive fusion fuel tritium should be minimal, and the irradiation of the material with $14 \mathrm{MeV}$ neutrons from the fusion reaction should not produce long-lived radioactive transmutation products. One such material is tungsten. However, it has the drawback that it can be severely damaged by crack formation or local melting in case of excessive power loads [1, 2]. Therefore, it has been suggested that liquid metal PFCs may be an alternative to solid divertor targets (see [1] and references therein). Apart from lithium and low-melting eutectic alloys, also tin $\mathrm{Sn}$ ) has been suggested, since it promises a very low vapor pressure from the melting point at $505 \mathrm{~K}$ up to about $1000 \mathrm{~K}$, as well as an endothermic solubility for hydrogen isotopes from the gas phase $[1,3]$.

So far, experiments and computer simulations of liquid tin as plasma-facing material have focused mainly on the heat load handling capability and erosion of prospective components (see, e.g., [1, 2, 4]). Data on hydrogen isotope uptake in tin due to plasma irradiation is still very sparse; so far we only found three references for pure $\operatorname{Sn}[5,6,7]$. In fact, even fundamental data on hydrogen 
isotopes in tin, such as diffusion coefficient and solubility, are extremely sparse and also conflicting (see [3] and references therein). We therefore started a systematic investigation of deuterium (D) retention and release for solid and liquid tin exposed to a low-temperature D plasma with an ion flux of $\sim 10^{20} \mathrm{D} / \mathrm{m}^{2} \mathrm{~s}$ and ion energies well below the sputtering threshold of $\mathrm{S} n$ at different temperatures and $D$ fluences. In particular, precise temperature control of the sample allowed the exposure of $\mathrm{Sn}$ just $10 \mathrm{~K}$ below and above the melting point in order to study in detail the effects of the phase transition from solid to liquid. The experiment was also designed to assess possible chemical erosion of $\mathrm{Sn}$, i.e., due to the formation of metastable stannane gas $\left(\mathrm{SnD}_{4}\right)[8]$.

In this article, we first present details of our experimental procedures and analysis methods. We then discuss the influence of the D plasma exposure conditions on the Sn surface morphology, D depth profile, thermal release spectrum of D-containing molecules, and on the net erosion rate and nearby re-deposition of Sn.

We found very different D uptake behavior of Sn exposed to D plasma at 300, 495 and $515 \mathrm{~K}$, ranging from extremely high $\mathrm{D}$ concentrations in a narrow subsurface region over deep penetration accompanied by formation of a thick, sponge-like layer, to the rapid formation of macroscopic gas bubbles underneath liquid tin. These different phenomena are also reflected by strongly different $D$ release behavior. In addition, we found evidence of chemical erosion of solid Sn by D plasma. Weight loss of the samples increased in good approximation linearly with $D$ ion fluence despite ion energies substantially below the sputtering threshold. The erosion rate strongly dropped from 300 to $495 \mathrm{~K}$, while in the liquid state at $515 \mathrm{~K}$, it sharply increased again due to ejection of a large number of $\mathrm{Sn}$ micro-droplets. In addition, an unexpected strong change in the wetting behavior of Sn on W was observed. We propose the hypothesis that at least some of the effects with respect to surface morphology and $\mathrm{D}$ retention are related to the chemistry of $\mathrm{Sn}$ and D, i.e., the formation and decomposition of stannane or maybe also its radicals. This is also reflected by correlated changes of the erosion rate and mechanism.

\section{Experiment details}

\subsection{Sample preparation and crucible design}

As base material, Sn pellets with $99.999 \%$ nominal purity purchased from Kurt J. Lesker, Inc., were used. Prior to plasma exposure, the $\mathrm{Sn}$ was washed in $10 \%$ aqueous $\mathrm{HCl}$ solution to remove contamination and, particularly, Sn oxides. The Sn pellets were then pre-molten into their crucibles using a high-vacuum oven, whose interior is made entirely from molybdenum (Mo). The samples were slowly heated with a ramp of $1 \mathrm{~K} / \mathrm{min}$ to allow the release of possible gas pockets, and then held at $523 \mathrm{~K}$ for 2 hours. Afterwards, they were cooled back down to room temperature with a ramp of $5 \mathrm{~K} / \mathrm{min}$.

The crucibles (see Figure 1) were milled from copper due to its excellent heat conductivity and machinability, and coated with about $2 \mu \mathrm{m}$ of tungsten (W) by magnetron sputter deposition, since $\mathrm{W}$ is practically immiscible with both $\mathrm{Sn}$ and $\mathrm{Cu}$. It thus acts as an effective separator between $\mathrm{Sn}$ and $\mathrm{Cu}$, which would otherwise form an alloy. Preliminary melting tests showed that this coating was stable up to about $540 \mathrm{~K}$. The crucible design was chosen such that it also allowed precise weight measurements before and after plasma exposure in order to assess possible $\mathrm{Sn}$ net erosion, e.g., by stannane $\left(\mathrm{SnD}_{4}\right)$ formation (see, e.g., [8]) or $\mathrm{Sn}$ droplet ejection [7]. In addition, a Mo witness sample was mounted next to the Sn-filled crucible in order to assess potential nearby re-deposition of eroded Sn. 

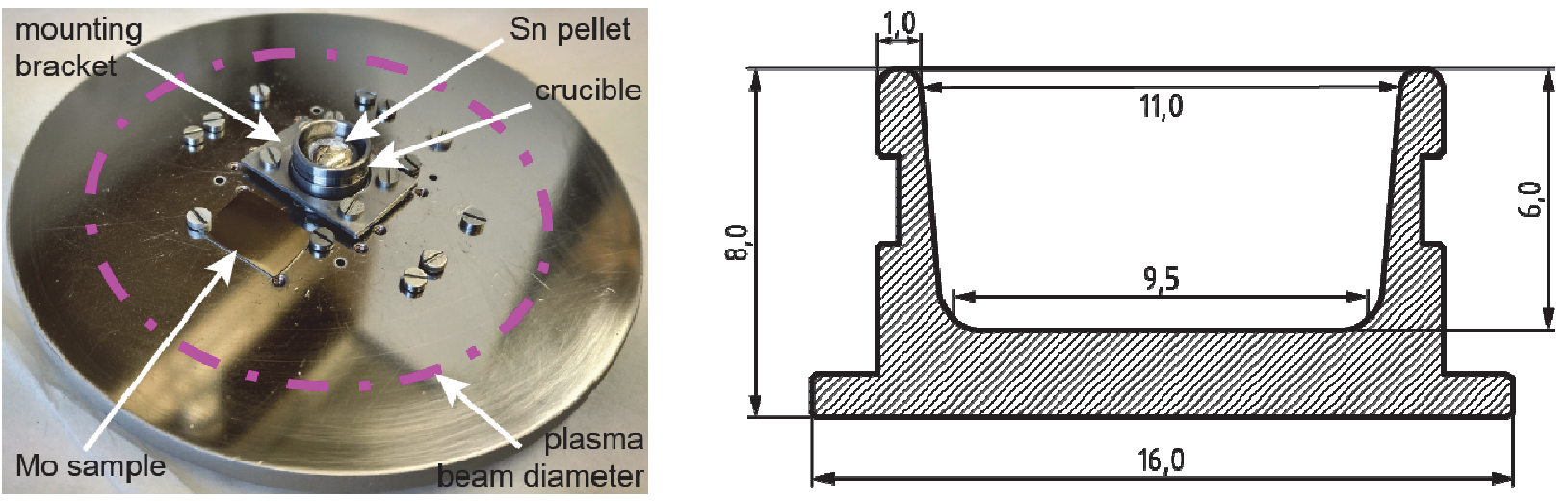

Figure 1: Crucible filled with pre-molten Sn mounted on the W-coated sample holder for plasma exposure (left side). The crucible is made from copper and coated with $2 \mu \mathrm{m}$ of $W$ to prevent alloying of $\mathrm{Cu}$ and $\mathrm{Sn}$. It is fixed to the holder with a mounting bracket made from W-coated stainless steel using Mo screws. The Mo witness sample is used to investigate Sn re-deposition. The right-hand panel shows a cross-section sketch of the crucible including dimensions in $\mathrm{mm}$.

For reliable thermal desorption spectroscopy measurements, it is necessary to remove the $\mathrm{Sn}$ from the crucibles after plasma exposure. This is facilitated by a slightly conical interior of the crucibles together with the typically relatively poor wetting of $\mathrm{W}$ by $\mathrm{Sn}$ at the temperatures investigated here. The necessity to remove the $\mathrm{Sn}$ from the crucible also prohibited the use of a so-called "capillary porous system" (CPS). Such set-ups retain the tin melt inside a porous matrix (e.g., a fine tungsten mesh) by capillary forces. The CPS arrangement is also proposed to hinder, e.g., melt splashing due to magneto-hydrodynamic forces that would occur in a fusion reactor or a high-flux linear plasma device $[1,2,9]$ by subdividing the melt into small compartments. Due to the orders of magnitude lower currents and weak magnetic fields in the plasma source used here, a CPS was also considered unnecessary for the experiments presented in this article.

\subsection{Plasma exposure}

The Sn samples were exposed in the electron-cyclotron-resonance plasma source "PlaQ" [10, 11, 12]. It delivers about $94 \% \mathrm{D}_{3}{ }^{+}$ions, as well as minor amounts of $\mathrm{D}_{2}{ }^{+}$and $\mathrm{D}^{+}$(3\% each). This means that the actual deuteron flux is about 3 times the ion flux. Correspondingly, the dominant ion energy can be estimated by $\left(\mathrm{V}_{\text {bias }}+\mathrm{V}_{\text {plasma }}\right) / 3$, whereas $\mathrm{V}_{\text {bias }}$ is the applied bias voltage. The plasma potential $\mathrm{V}_{\text {plasma, }}$ as determined by Langmuir probe measurements, is typically about $5 \mathrm{~V}$ (see Figure $\mathrm{S} 1$ in the supplementary material). Both potentials are specified relative to ground potential. In addition, the sample is hit by a flux of low-energy neutral $D$ atoms, which is estimated to be of the order of 100 times higher than the deuteron flux originating from ions. Recent measurements using a retarding field analyzer and a moveable Langmuir probe measuring the ion saturation current $\mathrm{j}_{\text {sat }}$ indicated that the ion flux was about $20 \%$ higher than reported in $[11,12]$ after exchanging the $\mathrm{Al}_{2} \mathrm{O}_{3}$ window through which the microwave radiation is transmitted into the vacuum vessel, i.e., the flux is now about $1.2 \times 10^{20} \mathrm{D} / \mathrm{m}^{2} \mathrm{~s}$. Repeated Langmuir probe profiles showed only minimal variations between the individual plasma discharges during which Sn samples were exposed. The plasma profiles were nearly flat in the region where the samples were exposed (see Figure 2). 


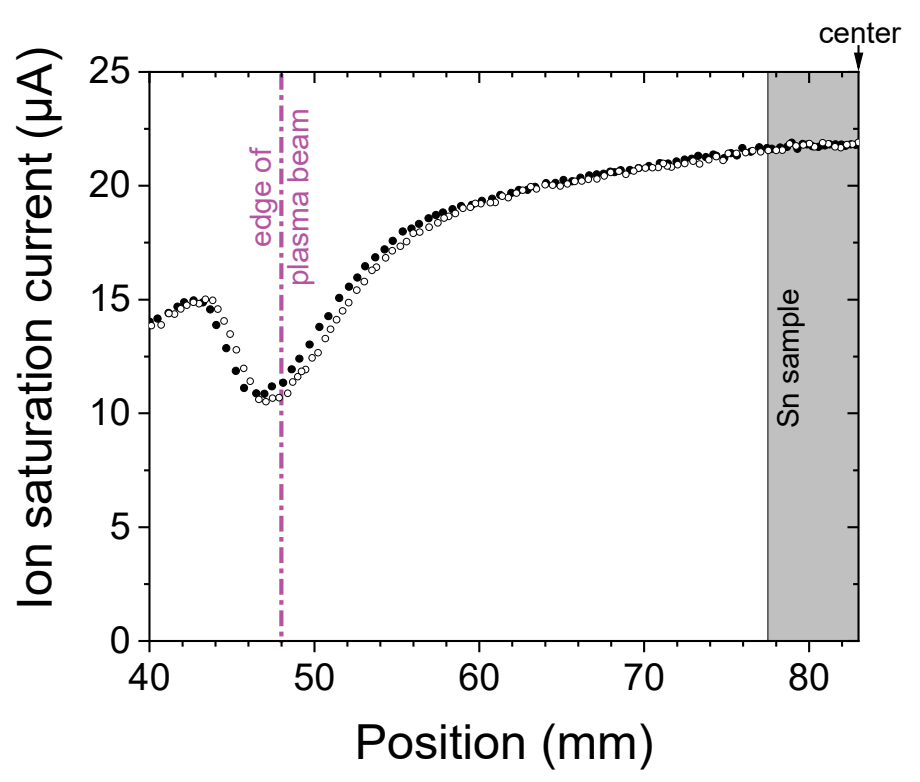

Figure 2: Langmuir probe profile of the ion saturation current through half of the cylindrically symmetric plasma beam, acquired using a probe bias of $-25 \mathrm{~V}$. The sample holder was also biased to $-25 \mathrm{~V}$ during the measurement. The edge of the visible plasma beam is located at the local minimum at about $x=48 \mathrm{~mm}$ (see also Figure 1). The Sn sample is mounted in the center at position $x$ $=83 \mathrm{~mm}$ (see also Fig. 1). The closed symbols represent the scan from the edge of the plasma to the center, and the open symbols were recorded on the way back out. The slight hysteresis between the two curves is due to the finite measurement speed during a continuous movement of the probe.

For exposing the $\mathrm{Sn}$ samples, we chose to apply a bias voltage of $-25 \mathrm{~V}$ to the sample holder, which results in a maximum ion energy of about $30 \mathrm{eV}$ for the small fraction of $\mathrm{D}^{+}$ions in the plasma. Even this maximum $D$ ion energy is substantially below the sputtering threshold of $D$ on $S n$ of at least $50 \mathrm{eV}$ according to calculations using SDTrimSP 6.0 [13] (see also supplementary S3 and S4). However, this small bias voltage improves the ion flux and stabilizes the flat plasma profile shown in Fig. 2 . In addition, it allows qualitatively monitoring the stability of the ion flux by measuring the current onto the sample holder. The deuterons originating from the majority ion species $\mathrm{D}_{3}{ }^{+}$ correspondingly have a kinetic energy of about $10 \mathrm{eV} / \mathrm{D}$. According to SDTrimSP 6.0 [13] assuming this projectile energy, the mean implantation range of $D$ into $S n$ is about $1.8 \mathrm{~nm}$ (see supplementary S2), and the reflection yield of $D$ on $S n$ at the ion energy of about $10 \mathrm{eV} / \mathrm{D}$ (for $\mathrm{D}_{3}{ }^{+}$) is about $65 \%$. I.e., only about $35 \%$ of the incident ions are actually implanted into the sample, and all other ions are backscattered before they come to rest in the Sn. Please note that the exact, quantitative validity of binary collision approximation (BCA) codes such as SDTrimSP become questionable at energies in the $\mathrm{eV}$ range. However, comparing BCA calculations with molecular dynamics (MD) simulations for lowenergy hydrogen or deuterium impinging on tungsten surfaces with various orientations [14-18], one finds an agreement typically within a factor of 2-3 for the implantation range, and also a reasonable agreement with respect to the particle reflection yield. Please note that the MD simulations exhibit substantial variations of a similar order of magnitude as the differences to BCA due to different crystal orientations, and even due to different interatomic potentials. In lack of published MD data for $\mathrm{H}$ isotope implantation into $\mathrm{Sn}$, we therefore consider our SDTrimSP calculations currently to be a reasonable estimate. Particularly for liquid Sn, crystallinity should not play any role at all. The density change of $\mathrm{Sn}$ from room temperature to $600 \mathrm{~K}$ is only about $5 \%$ [19], and thus considered negligible compared to the other uncertainties in estimating the implantation range and reflection yield of $D$ impinging on Sn. Because of these uncertainties, we also neglect the small contribution of higherenergy deuterons originating from $\mathrm{D}^{+}$and $\mathrm{D}_{2}{ }^{+}$. 
The sample temperature was stabilized by clamping the Sn-filled crucibles to the sample holder made from $\mathrm{W}$-coated copper. The temperature is feedback-controlled using a liquid thermostat (Julabo $\mathrm{HT} 350-\mathrm{CU}$ ) with high-temperature oil as the heat transfer fluid and a type-K thermocouple pressed into a conical hole on the back of the sample holder directly underneath the crucible. Melting of $\mathrm{Sn}$ was observed to begin at $500 \mathrm{~K}$. This value is in line with the literature value of $505 \mathrm{~K}$ and the rated accuracy of the thermocouple $( \pm 2 \% \pm 2 \mathrm{~K})$. Temperatures stated in this manuscript refer to the nominal temperature indicated by the thermocouple.

For the experiments reported here, a visible light camera system was installed at PlaQ. It consists of a Nikon DS-MV camera and a Computar macro-zoom lens. It allowed observing the Sn samples in situ during the plasma exposures and thus to document the time scales of $\mathrm{Sn}$ surface modifications by the plasma. In addition, an infrared camera (optris PI with $9^{\circ}$ optics; wavelength range 8-12 $\mu \mathrm{m}$ ) was also observing the samples.

Solid Sn samples were exposed to D plasma for $1 \mathrm{~h}, 24 \mathrm{~h}$ and $96 \mathrm{~h}$ at $300 \mathrm{~K}$ and $495 \mathrm{~K}$. These exposure times correspond to fluences of $4.3 \times 10^{23}, 1.0 \times 10^{25}$ and $4.1 \times 10^{25} \mathrm{D} / \mathrm{m}^{2}$, respectively. After the $495 \mathrm{~K}$ irradiation, the sample was cooled down to room temperature within about 15 minutes. Liquid $\mathrm{Sn}$ was exposed to $\mathrm{D}$ plasma for $1 \mathrm{~h}$ at $515 \mathrm{~K}$, and afterwards cooled down to room temperature within 15 minutes. The time between the end of plasma irradiation and re-solidification of the sample was about 1 minute. An additional sample, which was not used for measuring the $D$ retention but for a metallographical cross-section, was exposed at $515 \mathrm{~K}$ for $75 \mathrm{~min}$ (corresponding to $5.4 \times 10^{23} \mathrm{D} / \mathrm{m}^{2}$ ). This sample cooled slowly to the solidus point within $15 \mathrm{~min}$ in order to check for possible relaxation effects after the end of the plasma exposure, and later used for metallographic crosssectioning (see section 3.2).

\subsection{Microscopy}

The sample morphology before and after plasma exposure was routinely recorded using a Keyence VHX-6000 digital optical microscope. In addition, the samples were observed by scanning electron microscopy (SEM) using a Philips ESEM XL30 microscope. Cross-sections were prepared by focused ion beam (FIB) milling using $30 \mathrm{keV} \mathrm{Ga}^{+}$ions and observed in-situ using a FEI Helios NanoLab 600 microscope. An additional re-solidified Sn sample exposed at $515 \mathrm{~K}$ for 75 minutes was used for metallographic cross-sectioning. After embedding the complete sample with its crucible in clear epoxy resin, it was cross-sectioned using a diamond wire saw. The two halves of the cross-section were then polished first with abrasive paper, and then on a neoprene pad (Stuers MD-chem) using acidic alumina nanoparticle suspension (Struers OP-A) mixed with water and hydrochloric acid. The resulting cross-sections were investigated using the Keyence VHX-6000 digital optical microscope and additionally using an OLYMPUS LEXT OLS4000 confocal laser scanning microscope (CLSM).

\subsection{Nuclear reaction analysis}

D depth profiles in Sn were measured by analyzing the energy spectra of protons from the ${ }^{3} \mathrm{He}(\mathrm{D}, \mathrm{p})^{4} \mathrm{He}$ nuclear reaction at a set of incident ${ }^{3} \mathrm{He}$ energies of $690,800,1200,1800,2400,3200$, 3800,4500 and $6000 \mathrm{keV}$. The protons were counted using an annular detector (Type: Mirion LEC $300-2000 \mathrm{SPE}$ ) at a scattering angle of $175^{\circ}$ (area-weighted mean). The detector is covered by a circular slit mask with an inner radius of $4.25 \mathrm{~mm}$ and an outer radius of $10.0 \mathrm{~mm}$ at a distance to the sample surface of $88 \mathrm{~mm}$. The calibrated solid angle is $19.5 \mathrm{msr} \pm 0.5 \%$. The proton spectra were analyzed using the appropriate reaction cross section by Wielunska et al. [20] at $175^{\circ}$ scattering angle. The uncertainty of this cross-section lies between $4.2 \%$ and $5.9 \%$, depending on ${ }^{3} \mathrm{He}$ energy. 
For deconvolution of the measured data, we used the software packages NRADC [21] and SIMNRA $7.02[22,23]$ using SRIM-2013 [24] stopping powers. The deconvolution method used here relies on Bayesian inference of the most probable $D$ depth profile that would result in the measured proton energy spectra. It approximates the true depth profile as a set of slabs with constant concentration ("layers"), and searches for the configuration with the minimum number of layers that leads to an accurate reproduction of the measured data. For example, regions with flat concentration profiles and/or poor depth resolution are modelled as thick layers. Regions with steep gradients are represented by thinner layers, if the depth resolution of the nuclear reaction analysis is sufficient. The NRADC software also outputs the statistical uncertainty of the deconvolution, which was of the order of about 1-5\% for the measurements investigated in this article. Another potential source of statistical error is the mounting and positioning of the samples, since the Sn pellets were not flat, but roughly hemispherical. Re-measurement of two samples exposed at $495 \mathrm{~K}$ indicates this uncertainty could be up to about $10 \%$. In addition, the accuracy of the NRA measurements is affected by the systematic uncertainty of the current measurement of the ${ }^{3} \mathrm{He}$ beam, which is needed to integrate the total dose applied to the sample. This uncertainty is about $3 \%$. Typically, the combined systematic uncertainty of current measurement, detector solid angle and nuclear reaction crosssection dominates over the statistical error of the deconvolution, which is of the order of about 1-5\% for the measurements presented in this article.

\subsection{Thermal desorption spectroscopy}

For acquiring thermal desorption spectra, all Sn samples exposed to plasma below the melting point were removed from the crucibles used during plasma exposure. The Sn pellets were placed onto a boat made from Mo foil with a K-type thermocouple welded to its bottom side. The boat was placed in the glass tube of the "TESS" set-up [25]. A temperature ramp of $3 \mathrm{~K} / \mathrm{min}$ up to $873 \mathrm{~K}$ was programmed for the tubular oven around the glass tube. With some delay, the samples followed this temperature ramp, up to a maximum temperature of $865 \mathrm{~K}$. At the melting point ( $505 \mathrm{~K}$ ), the sample temperature stagnated until the sample was completely molten, as it is typical for a first-order phase transition. The accurate reproduction of the melting temperature also allowed us to confirm the accuracy of the temperature measurement in our TDS experiments. The vacuum before starting a TDS experiment was typically in the $10^{-8} \mathrm{mbar}$ range in the glass tube and of the order of $10^{-9} \mathrm{mbar}$ in the main chamber of TESS, where the mass spectrometer is located. In order to capture possible fast transients of $D_{2}$ release, e.g., at the melting point, two quadrupole mass spectrometers (QMS) were always used in parallel. Both mass spectrometers are mounted at the main chamber of TESS. One instrument (Balzers DMM422) was set to record the mass channels 1-4, 12, 14, 16-20, 27-29, 32 and 44 amu with a cycle time of approximately 14.2 seconds and provided an overview over various gases released from the sample. The second mass spectrometer (Hiden DLS20) was set to the lowresolution mode and recorded only mass 4 with a cycle time of about $200 \mathrm{~ms}$. The temperatures of oven and sample as well as the pressure in the glass tube and in the main chamber were recorded by the mass spectrometer hardware to have the same time stamp. We calibrated the sensitivity of both mass spectrometers using a LACO calibrated $D_{2}$ leak valve, whose flow has a relative uncertainty of $4.6 \%$ according to the manufacturer. The sensitivity for HD molecules (Balzers DMM422 only) was additionally determined by recording the flow of HD from a calibrated volume through an orifice, which was in turn cross-calibrated against the LACO $D_{2}$ leak valve. To assess the statistical uncertainty of the Balzers DMM422, we compared the calibration factors acquired at the end of each of the TDS measurements and found a standard deviation of $1.1 \%$. For the Hiden DLS20, we found a systematic drift of the sensitivity throughout the measurement campaign. An overnight measurement with the calibrated leak valve open revealed that the drift rate was of the order of $0.81 \%$ per hour. For typical 
durations of the individual measurement sessions of about 5.5 hours, with the calibration measurement at the end, this results in a total sensitivity drift of up to about $4.5 \%$ during each measurement. We consider this as an additional uncertainty of the total $D$ release during TDS measured with the Hiden DLS20. Finally, in order to compare the total amounts of $D$ release during TDS with $D$ retention measured by NRA, the effective surface area of the samples needs to be known. For this, we considered the projected area of the Sn pellets, i.e., the area seen by the impinging ions from the plasma. This projected area is about $83 \pm 3 \mathrm{~mm}^{2}$.

In addition to the uncertainties discussed above, TDS measurements can be affected by the storage of the samples between plasma exposure and analysis. Outgassing or isotope exchange can reduce the amount of $D$ retained in the sample, and oxidation of the sample surface can lead to the partial release of $D$ not as $D_{2}$, but in the form of heavy water molecules ( $H D O$ and $\left.D_{2} O\right)$, which are very difficult to accurately quantify. To prevent the latter issue, all samples were stored in vacuum exsiccators at a pressure between 2 and $7 \mathrm{mbar}$ after plasma exposure and only exposed to air for brief periods if necessary for analysis. Indeed, we found no indication of heavy water release from Sn for the samples investigated here. A detailed discussion of the molecules released during TDS can be found in section 5. The storage time between plasma exposure and TDS was between 15 and 67 days, for technical reasons and because all other analyses needed to be performed before TDS (a detailed table can be found in Supplementary S8). The potential outgassing of the samples during storage was estimated by comparing NRA depth profiles of two samples acquired shortly after plasma exposure, and again about 2 months later (see section 4.1). The potential storage loss appeared to be $10 \%$ or less.

\subsection{Sn weight loss and re-deposition measurements}

All pre-filled and pre-molten Sn samples were weighed in air before and after plasma exposure using a Sartorius MC21S microbalance. The following measurement scheme was applied for all samples. First, the lights in the room with the microbalance were turned on, since these were found to cause a room temperature rise of up to $2-3^{\circ} \mathrm{C}$. No air conditioning was used in this room because the air turbulence was found to be more detrimental to the weighing accuracy than slow temperature variations. After waiting at least 30 minutes until the room temperature had approximately stabilized, the automatic calibration function of the microbalance was executed. It was also necessary to compensate for varying buoyancy of the samples in air, which is caused by daily variations of temperature, air pressure and humidity. For this, a reference crucible filled with $\mathrm{Sn}$ and pre-treated like all other samples (except for plasma exposure) was measured at the beginning of each weighing session. The scale of such day-to-day variations was found be of the order of up to more than $10 \mu \mathrm{g}$. While this is small compared to the total weight of the Sn-filled crucibles of about $7.9 \mathrm{~g}$, it is not negligible compared to typical weight loss values. For precise weight measurements, weighing was repeated at least 5 and up to 7 times, depending on the variability of the individual weighings. After each weighing, the balance was unloaded, and, if necessary, re-zeroed. The average of these individual weight measurements was then taken as the actual weight of the reference sample. In between weighing sessions, the reference sample was stored in a vacuum exsiccator together with the other Sn samples. After determining the weight of the reference crucible, the same weighing procedure was then repeated for one or several crucibles to be exposed, or recently exposed to plasma. The apparent weight of a sample (i.e., the averaged value) was then finally corrected by multiplying it with the apparent weight of the reference crucible for the day and dividing it by the average of all reference measurements. 
To check for any potential influence of handling the samples and mounting them onto the PlaQ sample holder using the mounting bracket shown in Fig. 1, one sample was weighed, mounted and dismounted (without actually putting it into vacuum) and weighed again. The weights before and after the test mounting were $7907.8747 \pm 0.0008 \mathrm{mg}$ and $7907.8746 \pm 0.0010 \mathrm{mg}$, i.e., identical with a very high precision. This shows that our handling procedures indeed ensured a negligible influence on the weight measurements, and also the excellent reproducibility of the weighing itself.

The weight loss for any sample exposed to plasma was finally calculated subtracting the averaged, corrected weights before and after exposure. The uncertainty of the weight loss was estimated by Gaussian error propagation considering the uncertainties of both weighing sessions for the exposed sample as well as for the respective correction factors derived from weighing the reference sample.

Nearby re-deposition of $\mathrm{Sn}$ was investigated using the Mo witness sample mounted onto the sample holder next to the $\mathrm{Sn}$-filled crucible (see Fig. 1). The Mo samples were routinely measured by Rutherford Backscattering Spectroscopy (RBS) using ${ }^{3} \mathrm{He}^{+}$ions with an energy of $3200 \mathrm{keV}$. The detector used for this was a Canberra PD 25-10-500 PIPS detector with a nominal thickness of 500 $\mu \mathrm{m}$ and a nominal energy resolution of $10 \mathrm{keV}$. It was mounted at a scattering angle of $165^{\circ}$ in IBM geometry at a distance of $64.1 \mathrm{~mm}$ from the sample surface. The detector was covered by a slit mask of $1 \mathrm{~mm}$ width and $5 \mathrm{~mm}$ height. The measured detector solid angle was $1.108 \pm 0.038 \mathrm{msr}$. For $\mathrm{Sn}$ deposited onto Mo, a small but clearly recognizable signal can be distinguished already for about one monolayer $\left(\sim 10^{15} \mathrm{~cm}^{-2}\right)$ of $\mathrm{Sn}$, as SIMNRA $7.02[22,23]$ calculations (using SRIM-2013 [24] stopping powers) show (for measured example spectra as well as simulation results, see Supplementary S9). If signs of $\mathrm{Sn}$ re-deposition were found, the Mo sample was additionally investigated by electron microscopy using the Philips ESEM XL30 and by optical microscopy using the OLYMPUS LEXT OLS2000.

\section{Surface modifications}

\subsection{Solid Sn}

The different exposure conditions discussed in this article led to vastly different modifications of the sample surfaces: Plasma irradiation at $\mathbf{3 0 0} \mathbf{K}$ macroscopically caused a slight, gradual dulling of the originally shiny $\mathrm{Sn}$ sample surface (see animation in supplementary material). The optical changes were strongest within the first hour $\left(4.3 \times 10^{23} \mathrm{D} / \mathrm{m}^{2}\right)$, and nearly stagnated after about one day $\left(1 \times 10^{25} \mathrm{D} / \mathrm{m}^{2}\right)$. Microscopically, the surfaces showed roughening on length scales of a few microns, and height amplitudes of typically less than 1 micron (see Figure 3 ). The magnitude of roughening increased with the exposure time. For exposure times of $24 \mathrm{~h}\left(1.0 \times 10^{25} \mathrm{D} / \mathrm{m}^{2}\right)$ and $96 \mathrm{~h}\left(4.1 \times 10^{25}\right.$ $\left.\mathrm{D} / \mathrm{m}^{2}\right)$, the peaks and ridges of the roughness structure were usually crowned with small $\mathrm{Cu}$ - and Ferich nano-particles. The origin of these particles is presently unclear. In addition, small pores on the $100 \mathrm{~nm}$ up to $\leq 1 \mu \mathrm{m}$ scale could be found on images with higher magnifications. Focused ion beam (FIB) cross-sectioning revealed that these extended to a depth of about $1.5 \mu \mathrm{m}$. These pore structures were unstable and changed shape under irradiation with the electron beam or also with the ion beams of the microscope. It is plausible that such changes could be due to the heat load introduced by the strongly-focused electron beam, especially if it is scanned across sharp edges or thin skins of subsurface pores. The surface of the freshly-prepared FIB cross-section seemed particularly sensitive to electron beam irradiation. This may be due to the Ga implanted during FIB milling, since alloying $\mathrm{Sn}$ with Ga can reduce the melting point compared to pure $\mathrm{Sn}$ according to the binary phase diagram. The rapid changes of the cross-section surface during electron beam imaging also imply that most likely, the shape and number of pores visible in Figure $3 \mathrm{~b}$ may not accurately 
represent the original state of the material, although the qualitative picture should still be valid and is consistent with the surface images (Figure 3a) and NRA depth profiles (see section 4.1).
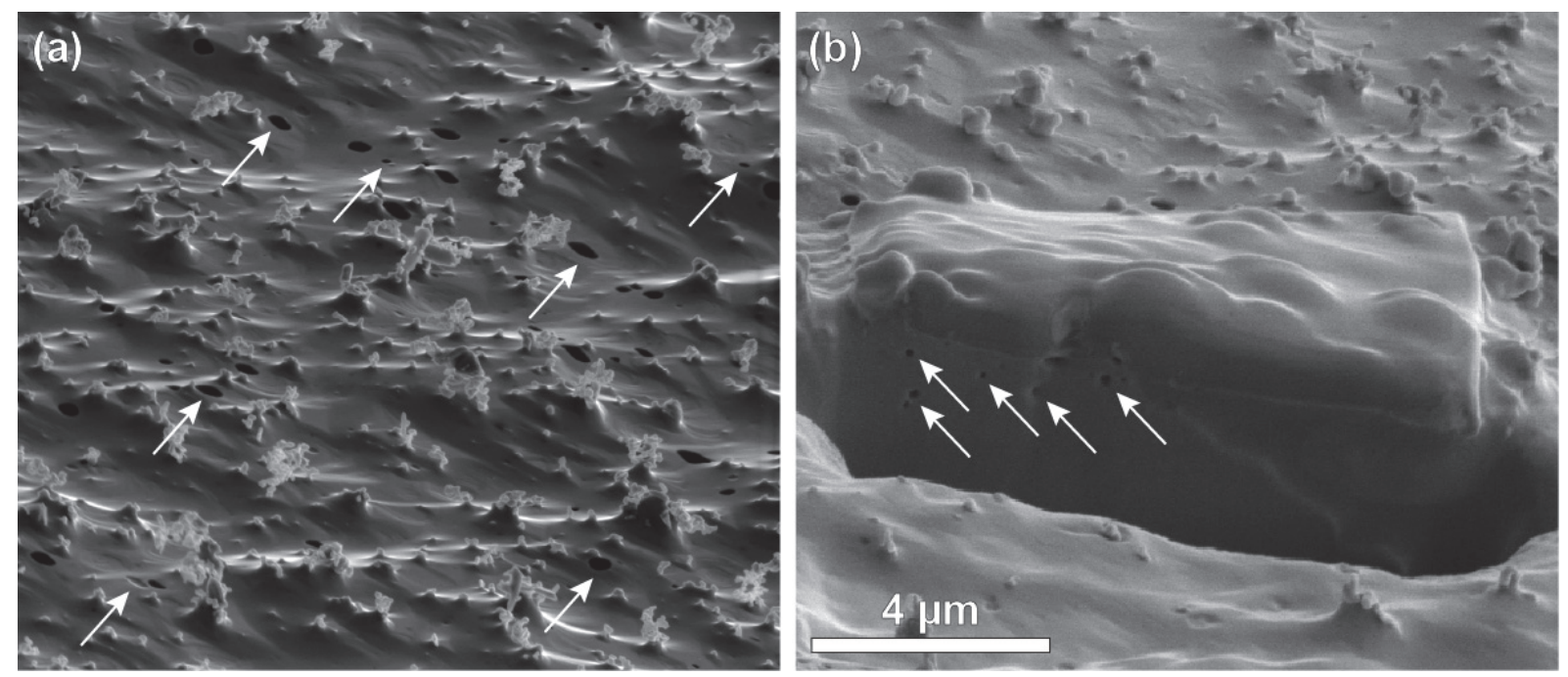

Figure 3: SEM images of surface (a) and FIB cross-section (b) of Sn exposed to D plasma for $96 \mathrm{~h}$ $\left(4.1 \times 10^{25} \mathrm{D} / \mathrm{m}^{2}\right)$ at $300 \mathrm{~K}$. In both images, the viewing angle is $52^{\circ}$ relative to the surface normal. This leads to a viewing angle of $-38^{\circ}$ for the cross-sectional surface in panel (b). The magnification is identical for both panels. The surface shows dune-like roughening, with crests and ridges crowned by impurity particles rich in $\mathrm{Cu}$ and/or Fe. Small round pores are also visible on the surface. The crosssection image (b) shows small pores at depths up to the $\mu \mathrm{m}$ range. In both panels, some pores are indicated by arrows. Note that the pores were unstable under the SEM electron beam, particularly in the cross-section image, i.e., they changed their size or vanished over time (see also animations in supplementary material).

Plasma irradiation at $\mathbf{4 9 5} \boldsymbol{K}$ led to the formation of a thick, sponge-like and highly porous layer. Optically, the sample surface was dulled much more strongly than at $300 \mathrm{~K}$, and developed a visibly rough, velvet-like appearance particularly for longer exposure times. Scanning electron microscopy revealed a sponge-like layer that started out as a wavy surface with many open pores and craters and structure sizes of several $10 \mu \mathrm{m}$ (Figure $4 \mathrm{a}$ ). FIB cross-sectioning showed that this structure contained open as well as closed porosity and extended to about $20 \mu \mathrm{m}$ below the surface (Figure $4 \mathrm{~d}$ ). This is remarkable since the mean implantation range is only $1.8 \mathrm{~nm}$ according to SDTrimSP 6.0 [13] calculations (see section 2.2.). For increasing exposure time, the porous, yet mostly still compact layer developed into a filamentary structure of $\mathrm{Sn}$ walls and tendrils vaguely outlining the possible remains of large bubbles (see Figure $4 b, c$ and e). A sequence of FIB cuts revealed that filaments themselves also contained smaller closed pores (see video in supplementary material). At larger depths, also larger closed pores were found. The size of these pores is roughly comparable to those found in the bubble layer observed after $1 \mathrm{~h}$ exposure, but the depth of this layer now extended to over $100 \mu \mathrm{m}$. This trend continued for exposure up to 96 hours $\left(4.1 \times 10^{25} \mathrm{D} / \mathrm{m}^{2}\right)$ : On the surface, the sponge-like structures appeared rather similar to those observed after $24 \mathrm{~h}$, albeit possibly with slightly coarser filaments containing more small, apparently open pores. The thickness of the layer had again increased substantially and is estimated to be about 250 to $500 \mu \mathrm{m}$ based on SEM images of FIB cross-sections. It is difficult to state a precise value for the thickness because the surface is very strongly corrugated. Also, due to the large cutting depth, pores in the cross-section were to a large extent filled up by re-deposited material. Finally, the large thickness of the sponge-like layer formed during the $96 \mathrm{~h}$ exposure made it necessary to prepare the cross-section cut with a rather large aspect ratio in order to keep the cutting time within reasonable limits. This, in turn, made it 
necessary to observe the cross-section at a shallow viewing angle. Under these imaging conditions, the uncertainty of the exact viewing angle of the FIB cross-section also causes a substantial uncertainty in the measured depth. The very large thickness of the sponge-like layer forming under $D$ plasma irradiation at $495 \mathrm{~K}$ also became apparent in the in-situ video data, which showed slight but visible swelling of the Sn during the 96 hours exposure.

It is currently not fully clear how the sponge-like layers formed, and how they could grow to such large thicknesses. Considering the evolution of the layers with exposure time, the internal closed pores, as well as the aforementioned swelling, it appears implausible that the observed structures are simply the result of an etching process. Rather, the layers clearly appear to grow, which means that they should be formed either by re-deposition of material eroded from the bulk, or by "foaming", i.e., the growth, coalescence and rupture of gas bubbles nucleated in the Sn. Judging by the cross-section images (Figure $4 \mathrm{~d}$ and e) and the evolution of the structure with increasing plasma exposure time, "foaming" seems to be more likely. Furthermore, the structures visible at the surface of the sponge-like layer do not seem to get more filigree with increasing plasma exposure time (compare Figure $4 \mathrm{~b}$ and $\mathrm{c}$ ), so it is unlikely that the layer grows by further and further branching of the top layer of $\mathrm{Sn}$ filaments. Accordingly, the layer must have grown from the bottom up, i.e., new pores should nucleate in or close to the Sn bulk. At latest after 24 hours of plasma exposure at $495 \mathrm{~K}$, there is no longer a direct line of sight that would allow energetic ions to reach the bottom of the layer. Therefore, $D$ ions cannot be responsible for the oversaturation of the Sn bulk with $D$ necessary to nucleate and grow $D_{2}$-filled pores. The source must rather be $D$-containing neutrals. $D_{2}$ gas practically does not react with $\mathrm{Sn}$ under the conditions used here, leaving $\mathrm{D}$ atoms or $\mathrm{SnD}_{4}$ molecules (or related radicals), which could potentially reach the $\mathrm{Sn}$ bulk even after multiple collisions with the Sn tendrils. D atoms already have a high chemical potential, while the eventual decomposition of $\mathrm{SnD}_{4}$ could lead to the evolution of nascent $\mathrm{D}$, which also has a high chemical activity. Finally, these energetic neutral deuterium species then apparently lead to a sufficiently high concentrations of solute $\mathrm{D}$ within the bulk $\mathrm{Sn}$ to create further bubbles. If, furthermore, $\mathrm{Sn}-\mathrm{D}$ compounds (i.e., $\mathrm{SnD}_{4}$ or fragments thereof) would exhibit solubility in Sn under the conditions applied here, this could also lead to bubble nucleation in large depths by delayed decomposition of absorbed molecules into $\mathrm{Sn}$ and $\mathrm{D}_{2}$. In addition, the postulated processes of $\mathrm{SnD}_{4}$ decomposition or absorption would also compete with chemical erosion of $\mathrm{Sn}$, and therefore would be a plausible explanation why the erosion rate is substantially lower at $495 \mathrm{~K}$ than at $300 \mathrm{~K}$ (see section 6.1).

\subsection{Liquid Sn}

Plasma irradiation of liquid $\mathrm{Sn}$ at $\mathbf{5 1 5} \boldsymbol{K}$ caused rapid, dramatic changes of the behavior of the melt. Within about one minute, the originally hemispherical surface of the melt - due to the normally high surface tension of liquid $\mathrm{Sn}$ and its poor wetting of $\mathrm{W}$ at this temperature - suddenly became flat and exhibited almost perfect wetting of the crucible's W surface (see also supplementary S6). We suspect that this may be due to the reduction of surface oxides and possibly hydrogen coverage on the $\mathrm{W}$ coating, and possibly also on the $\mathrm{Sn}$ melt. Within the $1 \mathrm{~h}$ exposure time $\left(4.3 \times 10^{23} \mathrm{D} / \mathrm{m}^{2}\right)$, the melt then started to steadily rise to the top rim of the crucible (see video in supplementary material). Occasionally, rippling of the otherwise mostly quiescent Sn surface could be seen in the in-situ optical camera. A dust particle on the melt surface could be seen rotating with about $1 \mathrm{~Hz}$ with the infrared camera of $\mathrm{PlaQ}$, which hints at the $\mathrm{Sn}$ melt spinning with a similar frequency. One sample exposed at $515 \mathrm{~K}$ was quickly cooled down after 1 hour and later used for $\mathrm{D}$ depth profiling and thermal desorption spectroscopy. A second sample was exposed for about 75 minutes $\left(5.4 \times 10^{25} \mathrm{D} / \mathrm{m}^{2}\right)$ until the melt nearly overflowed from the crucible (see supplementary S6). This 
sample was cooled down slowly to the solidus point. The in-situ camera did not reveal substantially sagging of the Sn surface during cool-down, i.e., the observed structure was quite stable. The surface of the re-solidified Sn was strongly corrugated, and scanning electron microscopy revealed large flower-like structures as well as open porosity reaching deep below the surface (Figures $5 c$ and 6 ). For $\mathrm{Sn}$ that was molten and re-solidified without $\mathrm{D}$ plasma exposure (e.g., the initial samples), the surface had a very different appearance (compare Figure $5 \mathrm{a}$ and $\mathrm{b}$ ), and was overall much smoother. It is, at present, difficult to determine the reason of this surface corrugation after $D$ plasma exposure in the liquid state. One reason could be that during plasma exposure gas bubbles form in the melt, some of which rise again to the surface and burst. Some of these bubbles may also burst during resolidification, which could be an explanation for the observed open porosity. Indeed, we have indication of bursting bubbles from the in-situ video observation as well as from thermal desorption spectroscopy (see section 5 ). Ou et al. were recently also able to directly observe the formation and bursting of gas bubbles at the surface of D-plasma-exposed liquid Sn using a fast camera [7]. On the Mo witness plate (see Figure 1), we also found a large amount of Sn microdroplets, which most likely originated from these bubble bursts (for more details, see section 6 below). This kind of Sn spray was only found after D plasma exposure of liquid Sn. A metallographic cross-section of the entire sample (see section 2.4) revealed a huge cavity below the re-solidified $\mathrm{Sn}$ as well as one large pore and several smaller pores within the re-solidified melt (Figures $5 c$ and 6 ; for high-resolution images of both sample halves see supplementary $\mathrm{S5}$; a close-up of the large pore is shown in supplementary S7). Other possible reasons for the observed surface corrugation could be connected to the obviously changed wetting behavior and possibly also surface tension of the plasma-exposed melt. 


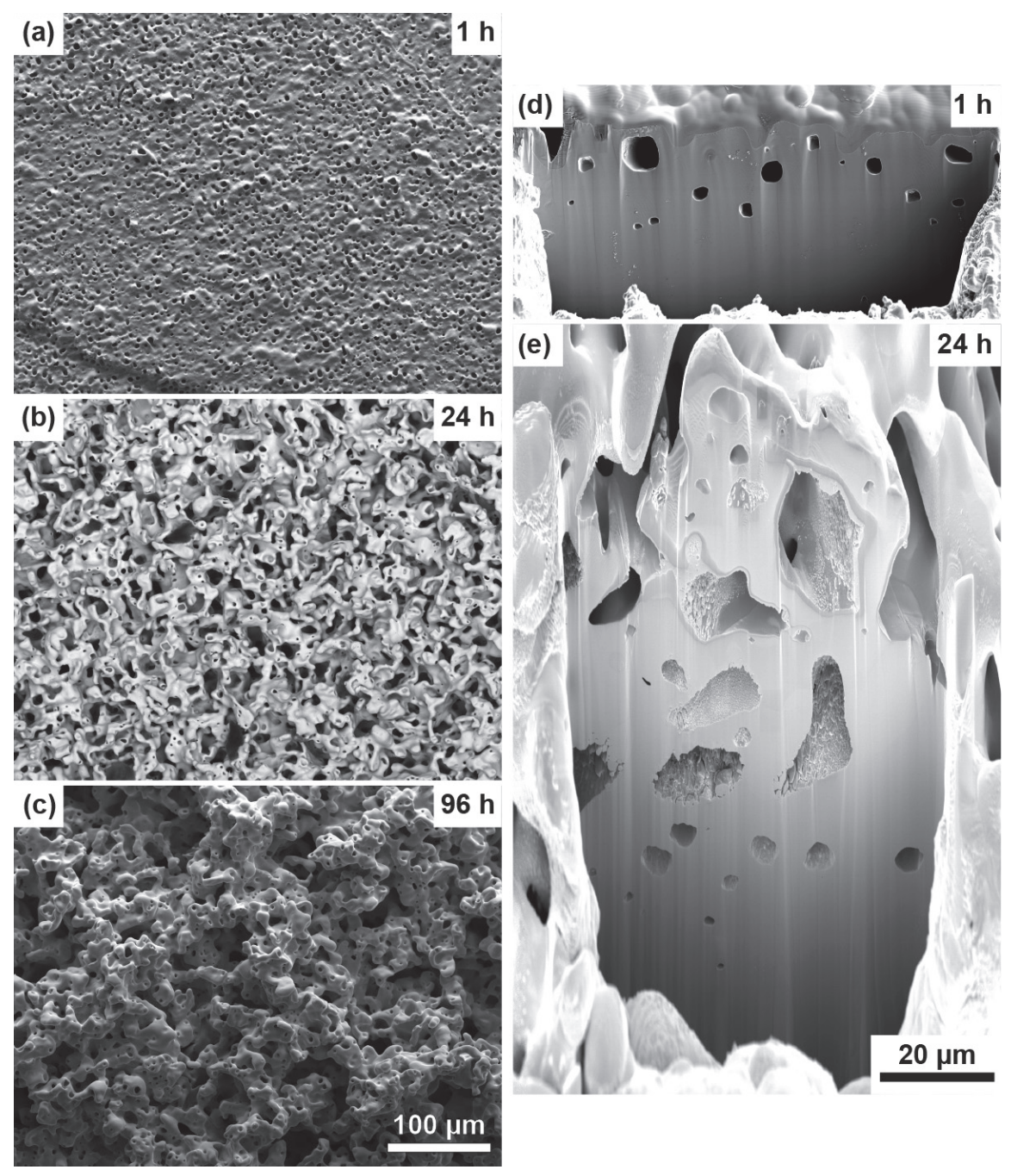

Figure 4: Evolution of Sn surfaces exposed to $D$ plasma at $495 \mathrm{~K}$ for increasing exposure times between $1 \mathrm{~h}$ and $96 \mathrm{~h}(\mathrm{a}-\mathrm{c})$, corresponding to fluences between $4.3 \times 10^{23} \mathrm{D} / \mathrm{m}^{2}$ and $4.1 \times 10^{25} \mathrm{D} / \mathrm{m}^{2}$, recorded by SEM. A sponge-like layer gradually develops and increases in thickness. The magnification is identical for all three panels. Panels (d) and (e) show SEM images of FIB crosssections corresponding to panels ( $a$ ) and (b), respectively. For the cross-section images, the original viewing angle was $38^{\circ}$ relative to the normal of the cross-section plane, but the $y$-axis was stretched in order to show the true aspect ratio of the structures visible on the cross-section plane. The scales are identical for these two panels as well. In both images, the Sn surface is covered by a protective Pt:C layer deposited prior to FIB cutting. The cross-sections reveal closed pores as well as pores that are connected to the surface. 


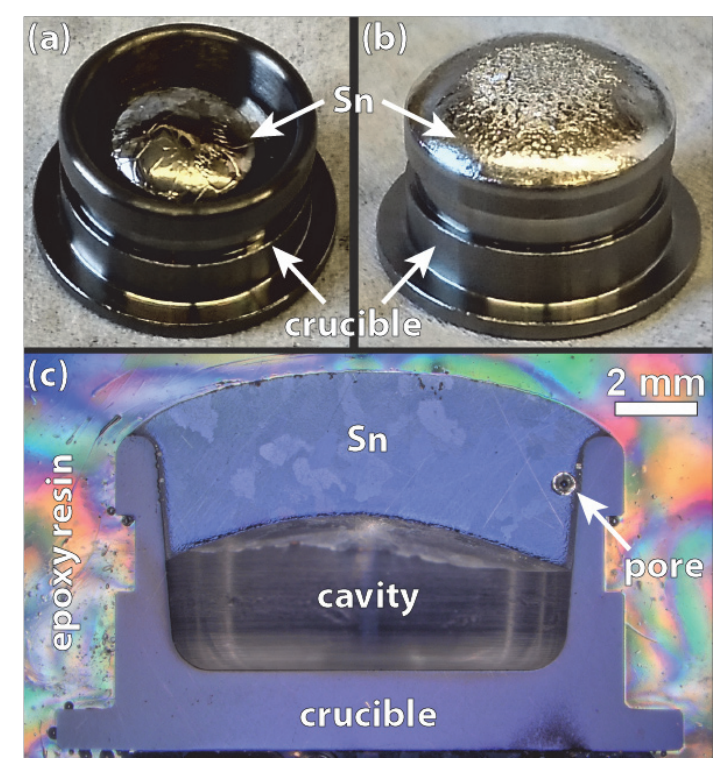

Figure 5: Sn sample used for D plasma exposure for $75 \mathrm{~min}$ at $515 \mathrm{~K}$. Panel (a) shows a photograph of the crucible before plasma exposure. The photograph in panel (b) was taken after removal from the plasma chamber. Panel (c) shows a metallographic cross-section of the Sn-containing crucible and reveals a large cavity below the re-solidified melt. A large pore within the melt can be seen at the right-hand side.

The large pore was in contact with the crucible wall, while the small pores were about evenly dispersed within the Sn. The slight etching caused by the acidic polishing agent made large grains with cloudy outlines visible in optical micrographs using coaxial illumination and a polarizing filter. As mentioned above, the cross-section also showed that the $\mathrm{Sn}$ melt had been pushed out from the crucible by a single large gas bubble underneath it (Figure $5 \mathrm{c}$ ). The bubble was tightly sealed by the Sn wetting the $\mathrm{W}$-coated crucible walls and had a similar volume as the piece of re-solidified Sn itself. The apex of the bubble was curved with a radius that was comparable to that of the plasma-exposed Sn surface. There was no obvious residue of $S n$ on the $W$ surfaces surrounding the large gas bubble below the re-solidified Sn, apart from one larger hemispherical Sn droplet on the bottom. The crosssection also showed that the wetting of the $W$ by the $S n$ melt was indeed excellent. E.g., at the crucible rim, where the Sn melt was just about to overflow, the contact angle between Sn and W was very small (see also supplementary $\mathbf{S 6}$ ).

Using the known mass of the $\mathrm{Sn}$ of $2.6 \mathrm{~g}$ and the geometry of the crucible ( $10 \mathrm{~mm}$ average inner diameter), it can be estimated that the pressure of the gas during plasma exposure must have been about $0.3 \mathrm{kPa}$ in order to levitate the $\mathrm{Sn}$ melt. This is 300 times more than the background $\mathrm{D}_{2}$ gas pressure of $1.0 \mathrm{~Pa}$ in the plasma chamber. The volume of the gas bubble together with the ideal gas law then yield that this corresponds to $10^{16}$ gas molecules trapped in the bubble. To estimate the amount of $D$ that should permeate through the Sn melt by diffusion alone, we use a simple flux balance model (see, e.g., [26]). A detailed derivation can be found in Appendix A. In short, the model yields that if diffusion-limited boundary conditions occur on both the plasma-exposed surface of the $\mathrm{Sn}$ and at the interface to the crucible, respectively to the gas-filled cavity, the ratio of permeating D to implanted $\mathrm{D}$ is proportional to the ratio of the implantation range to the thickness of the melt.

In our experiments, the mean implantation range of $D$ into $\mathrm{Sn}$ is about $1.8 \mathrm{~nm}$ (see section 2.2.). The geometrical thickness of the $\mathrm{Sn}$ melt is about $3.6 \mathrm{~mm}$ at the apex based on the metallographic crosssection of the sample. This yields an expected permeation ratio of the order of $5 \times 10^{-7}$. Using the 


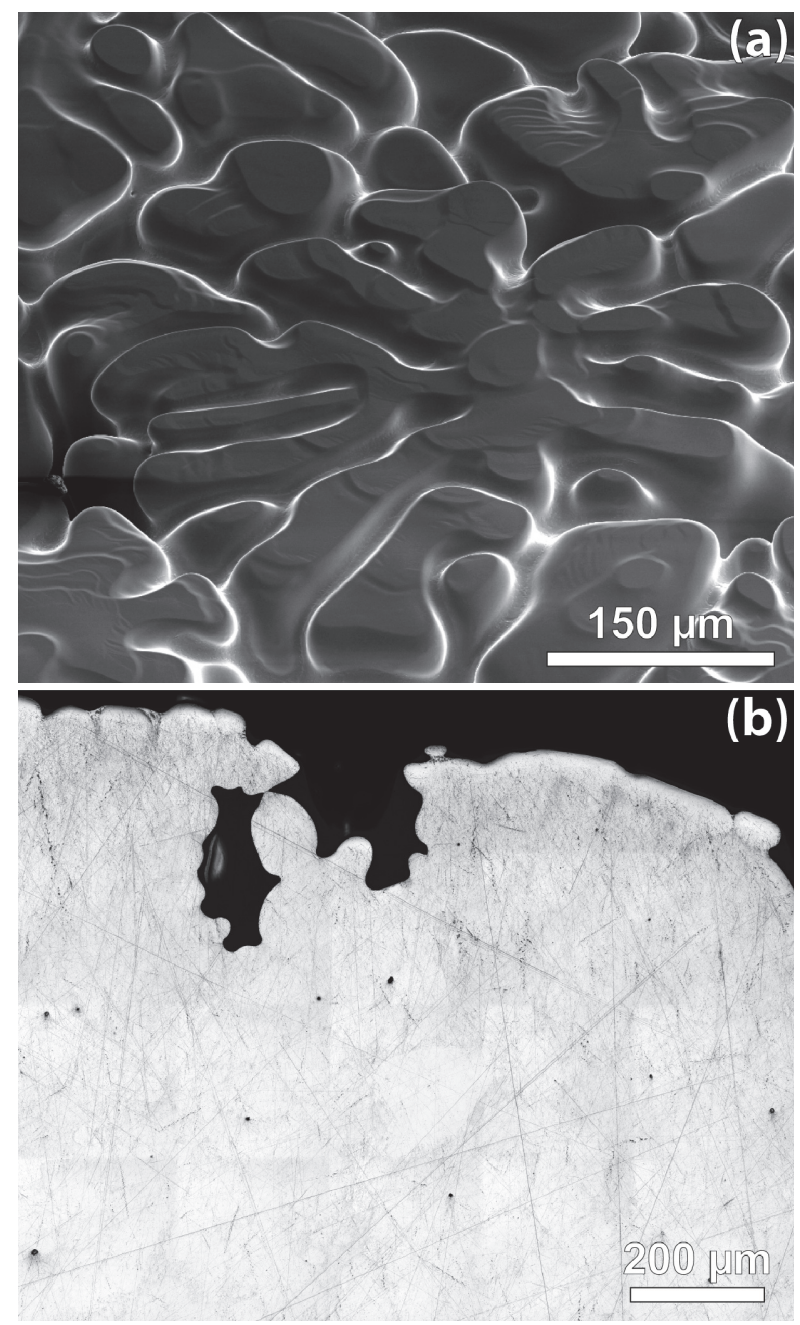

Figure 6: Re-solidified Sn after D plasma exposure for $75 \mathrm{~min}\left(5.4 \times 10^{23} \mathrm{D} / \mathrm{m}^{2}\right)$ at $515 \mathrm{~K}$. The sample surface is strongly corrugated and shows flower-like structures (panel (a), acquired by SEM under $52^{\circ}$ viewing angle). A metallographic cross-section (panel (b), acquired by confocal laser scanning microscopy) reveals open porosity reaching several $100 \mu \mathrm{m}$ below the surface, along with smaller pores dispersed in the re-solidified metal.

calculated reflection yield of $65 \%$ (section 2.2.), the ratio between the $D$ atoms (i.e., 2 times the number of molecules) estimated to be trapped in the large gas bubble $\left(10^{16} \mathrm{D}_{2}\right.$ molecules, corresponding to $\left.2.4 \times 10^{20} \mathrm{D} / \mathrm{m}^{2}\right)$ and the implanted particles $\left(5.4 \times 10^{23} \mathrm{D} / \mathrm{m}^{2} * 0.35=1.9 \times 10^{23} \mathrm{D} / \mathrm{m}^{2}\right)$ amounts to about $1.3 \times 10^{-3}$, and is therefore about 2500 times larger than what would be expected based on pure diffusion. One could argue that the re-emission of $D_{2}$ at the plasma-exposed surface is suppressed, i.e., the boundary condition might be recombination-limited instead of diffusion-limited, thus causing enhanced permeation into the bulk (for a detailed discussion of various boundary conditions for permeation, see also [26]). However, the D plasma effectively cleans the Sn surface, particularly from any oxides or organic contaminations. In addition, the steady bombardment with $D$ ions and atoms from the plasma can be assumed to rather stimulate than suppress $D$ desorption by providing recombination partners for effusing $D$, or by direct abstraction or self-sputtering of atomic D. In that light, we consider a recombination-limited boundary condition at the plasma-exposed Sn surface very unlikely. We also consider it unlikely that this large accumulation is due to the uptake and subsequent permeation via diffusive processes of neutral $D$ atoms from the plasma. According to a previous estimation [10], the neutral atomic $D$ flux is at least 10 times higher than the $D$ ion flux, 
but certainly not more than 1000 times. Assuming that most $D$ atoms are thermalized to the gas temperature by the time they reach the sample surface, they would adsorb at the Sn surface rather than penetrating it. Thus, an even smaller fraction than for the ions would contribute to permeation by diffusion. Let us assume finally that also $\mathrm{SnD}_{4}$ decomposition contributes to the source of $\mathrm{D}$, as postulated for the formation of the sponge-like layer at $495 \mathrm{~K}$. Considering the maximal effective chemical erosion yield measured in this work, which was found at $300 \mathrm{~K}$ (see section 6.1), is still much smaller than unity, we do not see how also $\mathrm{SnD}_{4}$ decomposition could lead to an orders of magnitude increase in the permeation flux through the $\mathrm{Sn}$ melt. Consequently, the amount of $\mathrm{D}$ transported into the large gas bubble cannot be explained by diffusion, and must therefore rather be facilitated by convection in the $\mathrm{Sn}$ melt, which could be induced, e.g., by magneto-hydrodynamic forces, possibly in combination with a (small) temperature gradient. The current density in our experiment was of the order of $1-2 \mathrm{~mA} / \mathrm{cm}^{2}$ and the magnetic flux density was of the order of $20 \mathrm{mT}$ at the location of the sample in our experiment. We plan a dedicated experimental campaign to study melt motion in our set-up in more detail, as well as its effect on deuterium retention. Gas bubbles rising to the surface due to buoyancy would also be a plausible drive for convection in the liquid Sn. Furthermore, a hydrogen plasma irradiation experiment of liquid GalnSn eutectic alloy subjected to forced $\mathbf{j} \times \mathbf{B}$ convection [27] showed reduced hydrogen recycling whenever the active convection was switched on. This is another indication that convection of a liquid metal can indeed enhance transport of hydrogen into larger depths. The exact nature, pattern and strength of the convection in our experimental arrangement is currently not clear, but as stated above, a dust particle on the surface could be observed to be spinning about once a second by the infrared camera observing the sample, which is a clear indication for melt motion.

\subsection{Comparison of gas bubble sizes and morphologies}

Comparing the Sn samples exposed to D plasma at 300, 495 and $515 \mathrm{~K}$, we observe the formation of gas bubbles under all three conditions, but with very different morphologies. At $300 K$, we find many small, isolated pores with roughly spherical or ellipsoidal shape close to the surface. The typical pore size is of the order of $0.1-1 \mu \mathrm{m}$ (Figure 3a). The pores are located close to the surface within a depth range of about $1.5 \mu \mathrm{m}$ (Figure $3 \mathrm{~b}$ ). At $495 \mathrm{~K}$, a thick, sponge like layer grows that contains a large number of pores. The sponge-like layer may be up to about $0.5 \mathrm{~mm}$ thick after $96 \mathrm{~h}$ of D plasma exposure. The pores can be round as well as irregular with complex 3-D shapes. The large, irregular pores are most likely formed by coalescence of smaller pores and are often connected to the surface. It seems that towards the bottom of the layer, pores rather tend to be small and round, while towards the top, the volume fraction of large, irregular pores dominates. However, there are still also smaller, round pores embedded in the Sn bridges between the large pores. The structures evolve with fluence, starting from mostly round pores and developing into the complex 3-D structures that eventually comprise a sponge-like layer hundreds of $\mu \mathrm{m}$ thick (compare Figures $4 \mathrm{~d}$ and e). At $\mathbf{5 1 5} \boldsymbol{K}$, i.e., in the liquid phase, we find small, round pores about evenly dispersed throughout the resolidified melt on a metallographic cross-section (see Figures $5 \mathrm{c}$ and $6 \mathrm{~b}$ ). These pores have dimensions ranging from several $\mu \mathrm{m}$ to tens of $\mu \mathrm{m}$. In this metallographic cross-section, a large, nearly spherical pore of about $0.6 \mathrm{~mm}$ diameter was also found attached to the vertical crucible wall, and it seems plausible that more of these should exist on other locations not visible in this specific cross-section. At the surface, a strong corrugation with lateral dimensions of the order of several 100 $\mu \mathrm{m}$ exists. It is connected to irregularly-shaped pores that appear to be open to the surface and also span up to several $100 \mu \mathrm{m}$, both laterally and into depth (Figure 6). Finally, a huge gas-filled cavities resides at the bottom of the crucible which spans its entire diameter of $10 \mathrm{~mm}$ and is several $\mathrm{mm}$ high. This bubble hat pushed the melt to the top of the crucible during plasma exposure. It was 
presumably created by smaller bubbles that were dragged along by convection of the melt and finally precipitated at the bottom of the crucible. We were not able to observe any of these structures in FIB cross-sections, since their dimensions as well as the typical distances between them are too large. Overall, we see a general trend towards larger gas bubbles at higher temperatures, whereas of course the phase transition from solid $\mathrm{Sn}$ at $495 \mathrm{~K}$ to liquid $\mathrm{Sn}$ at $515 \mathrm{~K}$ is an additional factor that very likely influences the size, shape and density of the bubbles.

\section{D depth profiling by nuclear reaction analysis}

\subsection{Solid Sn}

As for the surface modifications, the $D$ depth profiles showed a strong variation with the exposure temperature (see Figure 7). Plasma exposure at $300 \mathrm{~K}$ resulted in a very high D concentration of up to more than 10 at. $\%$ at the surface, which steeply decayed to about 0.1 at.- $\%$ within about $0.5 \mu \mathrm{m}$ for all exposure times. For increasing exposure time, a tail deeper into the bulk developed. The total amount of $D$ found by NRA was $(6.1 \pm 0.3) \times 10^{20} \mathrm{D} / \mathrm{m}^{2}$ for $1 \mathrm{~h}$ exposure, $(9.5 \pm 0.4) \times 10^{20} \mathrm{D} / \mathrm{m}^{2}$ for $24 \mathrm{~h}$ and $(12.8 \pm 0.6) \times 10^{20} \mathrm{D} / \mathrm{m}^{2}$ for $96 \mathrm{~h}$. We do not assume that the small $\mathrm{Cu}$ - and Fe-rich particles found on the surface of this sample have a noticeable effect on the $D$ retention. First of all, they only cover a very small part of the total surface area. In addition, both Fe and $\mathrm{Cu}$ have a low solubility for hydrogen isotopes and are therefore not expected to contain a substantial amount of $D$. If anything, they should cause a minimal shadowing of the Sn surface.

After $D$ plasma exposure at $\mathbf{4 9 5} K$, the $D$ concentration showed a narrow local minimum at the immediate sample surface. After $1 \mathrm{~h}$ exposure time, the $\mathrm{D}$ concentration then rose to about 1.5 at.- $\%$ and decayed to about 0.1 at.-\% within the analysis range of about $22 \mu \mathrm{m}$, which corresponds approximately to the extension of the observed bubble layer (compare Figure 4d). Please note that depths indicated for NRA measurements on the sponge-like structures observed for D plasma exposure at $495 \mathrm{~K}$ correspond to dense, compact $\mathrm{Sn}$ equivalents. Due to the large porosity of the sponge-like layer, this cannot be easily translated into geometrical depth. Also, the exact shape of the depth profiles must be taken with caution. For larger exposure times, the $D$ depth profiles are approximately flat at concentrations in the range of 0.1 at.-\%, apart from the local minimum at the surface. With increasing exposure time, this local minimum appears to become more pronounced. This depletion zone at the sample surface may be due to the highly porous structure of the samples (see section 3.1), i.e., more and more pores close to the surface rupture with increasing exposure time, and release their $D_{2}$ gas filling. The highly porous structure most likely also makes the nearsurface zone of the sponge-like layer more susceptible to outgassing during cool-down to room temperature after the plasma exposures finished. One factor is the very large effective surface. Another aspect is that the thermal conductivity between the Sn bulk and the sponge-like filaments at the surface is probably strongly reduced. Since the samples are not heated by the plasma but through the sample holder, this would reduce the effective cooling rate at the sample surface and therefore promote outgassing. Radiative cooling would, of course, also be increased by the rough surface, but should still be only a negligible contribution to the total cooling rate around $\sim 500 \mathrm{~K}$. For all exposure times, the amount of $D$ found within the NRA analysis range is comparable at about $3 \times 10^{21} \mathrm{D} / \mathrm{m}^{2}$, but it is clear the depth profiles for $24 \mathrm{~h}$ and $96 \mathrm{~h}$ exposure time extend deeper, i.e., beyond the analysis depth accessible by NRA (see also section 5.3 ).

In order to assess potential losses of $D$ during the sample storage between plasma exposure and analysis, the samples exposed at $495 \mathrm{~K}$ for $1 \mathrm{~h}$ and for $24 \mathrm{~h}$ were investigated by NRA once directly after plasma exposure (on the same day respectively on the next day), and then again about 2 
months later (i.e., shortly before TDS). The acquired depth profiles were very similar. Slight shifts of both profiles were observed, which may be due to the re-mounting and re-positioning of the roughly hemispherical samples. In terms of the total amount of $D$, the sample exposed for $1 \mathrm{~h}$ showed a $3.5 \%$ higher $D$ inventory in the second measurement, while the sample exposed for 24 hours showed a $10 \%$ lower total $D$ inventory. Since the differences between both sets of measurements were small and unsystematic, we believe they represent an additional statistical uncertainty due to mounting and positioning of the samples rather than actual changes in the $D$ inventory.

\subsection{Liquid Sn}

After D plasma exposure at $\mathbf{5 1 5} \boldsymbol{K}$, the concentration of D found by NRA is at or below the detection limit, i.e., in good approximation vanishing. The few proton counts found in some measurements can be explained by a small amount of $D$ adsorbed at the sample surface. Although from these marginal signals no depth profiles can be reliably extracted, an NRADC deconvolution was performed in order to estimate an upper limit for the $D$ concentration in the sample. The deconvolution yielded a $D$ concentration of approximately $5 \times 10^{-5}$ at.- $\%$ if assuming a homogeneous $D$ distribution throughout the $\mathrm{Sn}$ (not shown in Figure 7). This is approximately the sensitivity limit of the NRA measurement, which is determined by the (small) background of about 0.01 counts/channel/ $\mu \mathrm{C}$ in the relevant energy range that appears at higher ${ }^{3} \mathrm{He}$ energies.

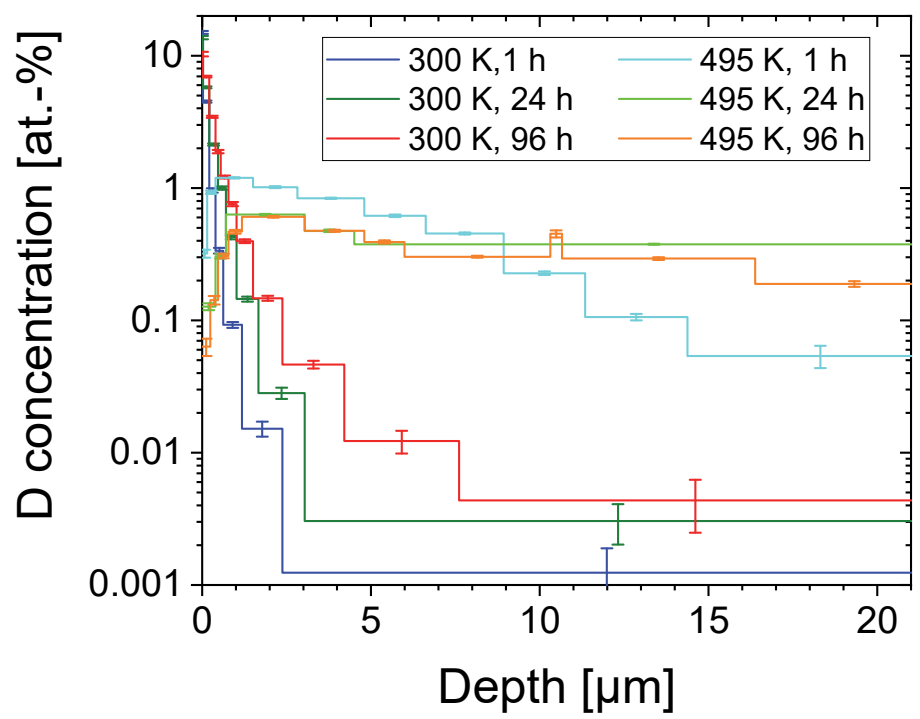

Figure 7: D depth profiles in Sn obtained by $D\left({ }^{3} \mathrm{He}, p\right)^{4} \mathrm{He}$ nuclear reaction analysis. The depth scale for these profiles corresponds to dense Sn equivalents. Note how $D$ depth profiles after plasma exposure at $300 \mathrm{~K}$ are strongly peaked at the surface, while for samples exposed at $495 \mathrm{~K}$, the profiles are much flatter and show a depletion zone at the surface.

\section{Thermal desorption spectroscopy}

\subsection{Solid Sn}

The dominant feature in the release spectrum of all samples exposed to $D$ plasma below the melting point of $S n$ was a massive release of mass 4 (attributed $D_{2}$ ) that began together with the melting of the Sn during TDS. The melting process was clearly observable in the temperature curve of the $\mathrm{Sn}$ 
sample: As soon as the melting point was reached, the sample temperature stagnated, while the oven temperature ramp continued. After about 750 seconds, the melting was finished, and the temperature of the sample rapidly increased again before asymptotically approaching the original ramp again. For the shortest exposure times, the $D_{2}$ release peak that occurred during this melting plateau was very narrow. It became broader (in time) and developed a tail with increasing exposure time, respectively $D$ ion fluence. For exposure at $\mathbf{4 9 5} K$, even a structure with multiple peaks developed, but in all cases, the mass 4 signal decayed quickly to the background level at the end of the melting phase.

We would like to point out that for all TDS spectra where the $D_{2}$ release is dominated by one or few sharp spikes (i.e., all samples exposed at 300 and $495 \mathrm{~K}$ ), the total amounts of $D_{2}$ release measured by the Balzers DMM422 mass spectrometer are unreliable. Because of the cycle time of about 14.2 seconds for this instrument (see section 2.5 ), the $D_{2}$ spikes could not be resolved properly. Since, on the other hand, there was practically no release of other D-containing molecules for these samples, we rely on the Hiden DLS20 for absolute quantification in these cases.

For D plasma exposure at $300 \mathrm{~K}$ (see Figure 8), mass 4 release is observed as soon as the heating ramp starts. At about $450 \mathrm{~K}$, a broad release peak was observed, which was in terms of total released amount of $D_{2}$ much smaller than the main release peak that occurred during melting. For all exposure times, the fraction of $D_{2}$ released before melting was about $1 / 3$ of the amount released during melting and decreased only very slightly with increasing exposure time. I.e., both the melting peak and the low-temperature peak increased about proportionally in their absolute integrated intensity.

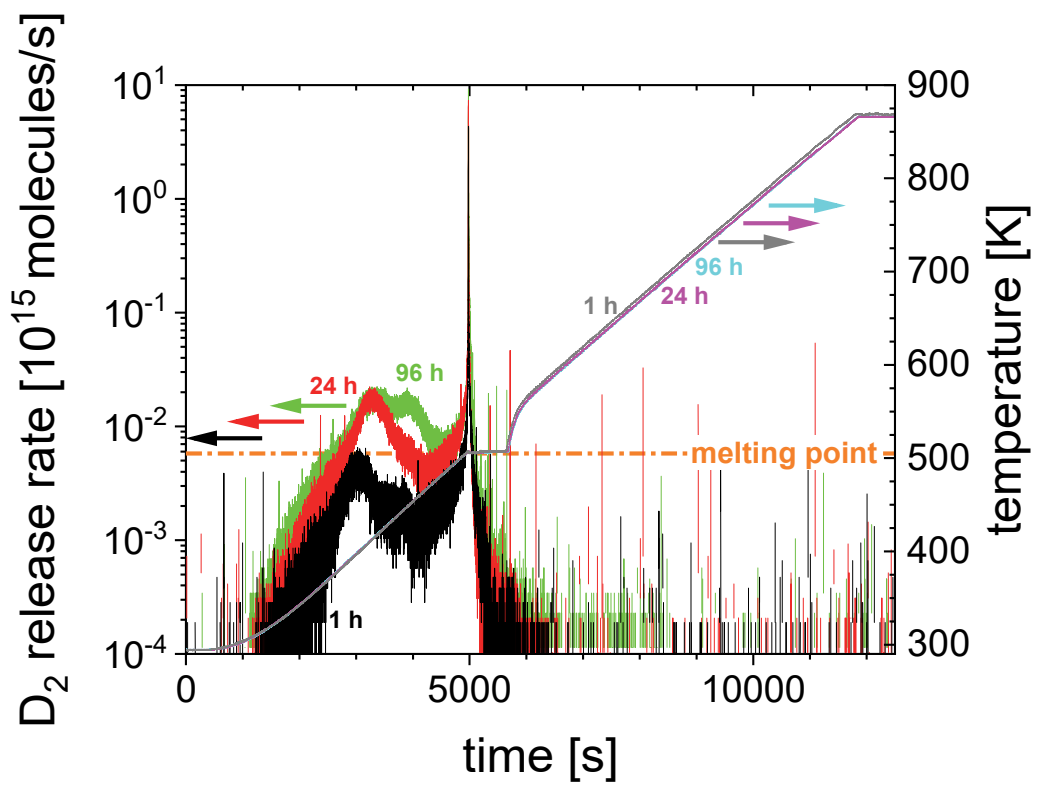

Figure 8: $D_{2}$ release spectra of $S n$ samples exposed to plasma at $300 \mathrm{~K}$ for various times (left $y$-axis). The spectra shown here were acquired using the Hiden DLS2O mass spectrometer. The time axis of the spectra has been synchronized to the main release peak, which occurs at the melting point. For reference, the temperature curve of all samples is shown (right y-axis). Note the distinct temperature plateau during melting. Besides the main release peak at the beginning of this plateau, a broad secondary release feature occurs at lower temperatures. After the main release peak, the signal quickly decays to background. The Balzers DMM422 spectrometer running in parallel did not record significant amounts of other molecules than $D_{2}$ released from the samples. 
After D plasma exposure at $495 \mathrm{~K}$ (see Figure 9), the release of $D_{2}$ started right at the beginning of the temperature ramp as well and gradually increased up to the melting point of tin. In this case, there was no distinguishable low-temperature peak. Accordingly, the fraction of $D_{2}$ released during melting was even higher, ranging from about $92 \%$ for $1 \mathrm{~h}$ plasma exposure up to $95 \%$ after 96 hours.

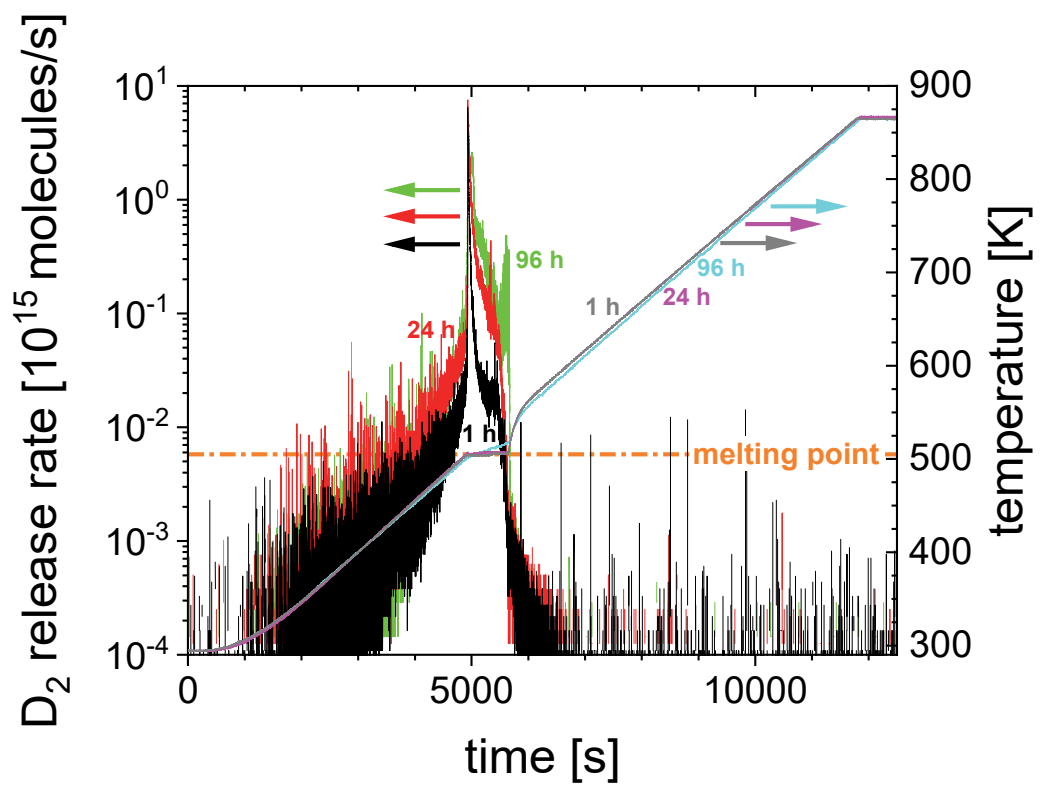

Figure 9: $D_{2}$ release spectra of $S n$ samples exposed to plasma at $495 K$ for various times (left $y$-axis). The spectra shown here were acquired using the Hiden DLS2O mass spectrometer. The time axis of the spectra has been synchronized to the main release peak, which occurs at the melting point. For reference, the temperature curve of all samples is shown (right $y$-axis). In contrast to Sn exposed at $300 \mathrm{~K}$, there is no secondary release peak at low temperatures. Instead, the main release peak is broader and splits into multiple peaks at long exposure times. Still, practically all $D_{2}$ is released by the end of the melting plateau. The Balzers DMM422 spectrometer running in parallel did not record significant amounts of other molecules than $D_{2}$ released from the samples.

For all samples that were exposed below their melting point and extracted from their crucibles before TDS, the release spectra were remarkably clean: Almost only mass $4\left(D_{2}\right)$ was released, with only a minor contribution from mass $3(\mathrm{HD})$, which was of the order of $1 \%$ of the mass 4 signal. Similarly, the mass 2 signal $\left(\mathrm{H}_{2}\right.$ and $\left.\mathrm{D}\right)$ was also very small, and could also be largely attributed to the dissociation of $D_{2}$ in the QMS ion source. There was almost no discernible signature of masses 18,19 and 20 (attributed to $\mathrm{H}_{2} \mathrm{O}, \mathrm{HDO}$ and $\mathrm{D}_{2} \mathrm{O}$, respectively) being released apart from a small peak in masses 19 and 20 that was synchronous to the main mass 4 release peak, and a slight increase of mass 18 with increasing temperature. We think it is plausible that the signals of masses 2, 3, 19 and 20 may actually largely originate from molecules being formed in the ion source of the QMS, by dissociative ionization of $D_{2}$ (yielding $D^{+}$at mass 2 ) or reactions with the residual gas yielding $\mathrm{HD}^{+}$, $\mathrm{HDO}^{+}$and $\mathrm{D}_{2} \mathrm{O}^{+}$. This assumption is backed by the observation that during calibration of the QMS using a $D_{2}$ leak valve, a very similar ratio of masses 3 and 4 was measured as during the main TDS release peak. The ratios of masses 2,19 and 20 to mass 4 were also of the same order of magnitude for the calibration and the actual TDS measurement, although here the intensities for masses 2, 19 and 20 during the calibration measurement lie just marginally above the background and are thus very uncertain. Due to the very small amount of $D$-containing molecules other than $D_{2}$ observed during TDS, these are neglected in the computation of the total $D$ inventory for the samples that were exposed below the melting point. 


\subsection{Liquid Sn}

The Sn sample exposed to $D$ plasma in the liquid state at $515 K$ could not be removed from its crucible due to the unexpected, very strong wetting of $\mathrm{Sn}$ on $\mathrm{W}$ caused by the influence of the plasma that was already described above (see, e.g., Figure 5 ). In order to estimate the $D_{2}$ release from the crucible, we therefore acquired the TDS spectrum of the emptied crucible belonging to the sample exposed at $495 \mathrm{~K}$ for $1 \mathrm{~h}$, which was the condition closest to the liquid $\mathrm{Sn}$ exposure. Exposure of an empty crucible at $515 \mathrm{~K}$ was considered unsuitable for determining this background, since the surface area of the W-coated crucible exposed to plasma would be substantially larger than that of a crucible filled with Sn. We therefore chose the emptied crucible of the Sn sample exposed at $495 \mathrm{~K}$ for $1 \mathrm{~h}$ as the best approximation for $\mathrm{D}$ release from the crucible for the liquid $\mathrm{Sn}$ sample. For this crucible, the plasma exposure conditions were as similar to the liquid $\mathrm{Sn}$ as possible. In addition, for $495 \mathrm{~K}$, we found the least amount of chemical erosion of $\mathrm{Sn}$, and also a negligible amount of redeposition onto the witness sample (for details see section 6). Therefore, we consider the amount of D co-deposited with $\mathrm{Sn}$ onto the crucible during the $495 \mathrm{~K}$ exposure to be negligible. The $\mathrm{D}_{2}$ release spectrum of the crucible exposed for $1 \mathrm{~h}$ at $495 \mathrm{~K}$ is shown in Figure 10 together with the release spectrum of the filled crucible exposed for $1 \mathrm{~h}$ at $515 \mathrm{~K}$. The two crucibles were not placed in the Mo boat used for the other $\mathrm{Sn}$ samples, but thermocouples were directly welded to their respective bottom surfaces. Basically, the crucible shows $D$ release in the form of a broad peak ranging from about 450 to $730 \mathrm{~K}$. The integral amount of $D_{2}$ released from the empty crucible corresponds to about $10 \%$ of the total $D_{2}$ release from the crucible filled with re-solidified $\mathrm{Sn}$ according to the Hiden DLS20 spectrometer.

The $D_{2}$ (mass 4) release from the Sn-filled crucible exposed for $1 \mathrm{~h}$ at $515 \mathrm{~K}$ consisted of two distinct features: One was a continuous signal that was similar in shape to the release from the emptied crucible, only with substantially higher intensity. This continuous release started at about $400 \mathrm{~K}$ and continued until at least $730 \mathrm{~K}$. On top of this signal, many sharp release spikes were observed. $\mathrm{D}_{2}$ release in the form of spikes started with a dense burst of spikes just when the melting started, but in contrast to all other samples, neither the continuous nor the spiky signal decayed as strongly when the melting was finished. Only a moderate peaking of the continuous signal was observed during the melting phase. When the Sn melted, the melt surface at first stayed roughly in the position where it had been during plasma exposure. For increasing temperature, however, the melt started to slowly rise from the crucible until its bottom was about level with the crucible rim. It then very suddenly dropped back into the crucible. This process was observed 3 times during the heating ramp and was each time correlated with a particularly large release spike of $D_{2}$ (and also other gases). On several occasions, also medium-sized spikes could be correlated to rippling of the otherwise calm and flat $\mathrm{Sn}$ melt surface. We attribute the rising of the Sn pellets to expanding gas inside a cavity at the bottom of the crucible similar to the one observed in the cross-sectioned sample exposed in the liquid state. Once the melt then rose above the crucible rim, part of that gas was released, thus reducing the gas pressure in the bubble levitating the $\mathrm{Sn}$ melt. The smaller spikes, on the other hand, are attributed to gas bubbles contained within the Sn melt and - apparently relatively sluggishly - rising to the surface due to buoyancy, where they burst. A slow movement of gas bubbles inside the melt would fit to the observation of large open cavity networks at the surface of the sample exposed at $515 \mathrm{~K}$ and then cross-sectioned: In this light, we interpret these structures as bubbles bursting at the moment of resolidification.

Based on preliminary $\mathrm{Sn}$ melting experiments in a vacuum oven, we have to assume that at temperatures around $573 \mathrm{~K}$, the W coating of the crucible started failing. The $\mathrm{Sn}$ melt increasingly 
attacked the crucible and alloyed with the $\mathrm{Cu}$, which also became obvious after removing the crucible from the TDS apparatus. Even in this temperature range, the deuterium release - mostly in the form of bursts - still continued.

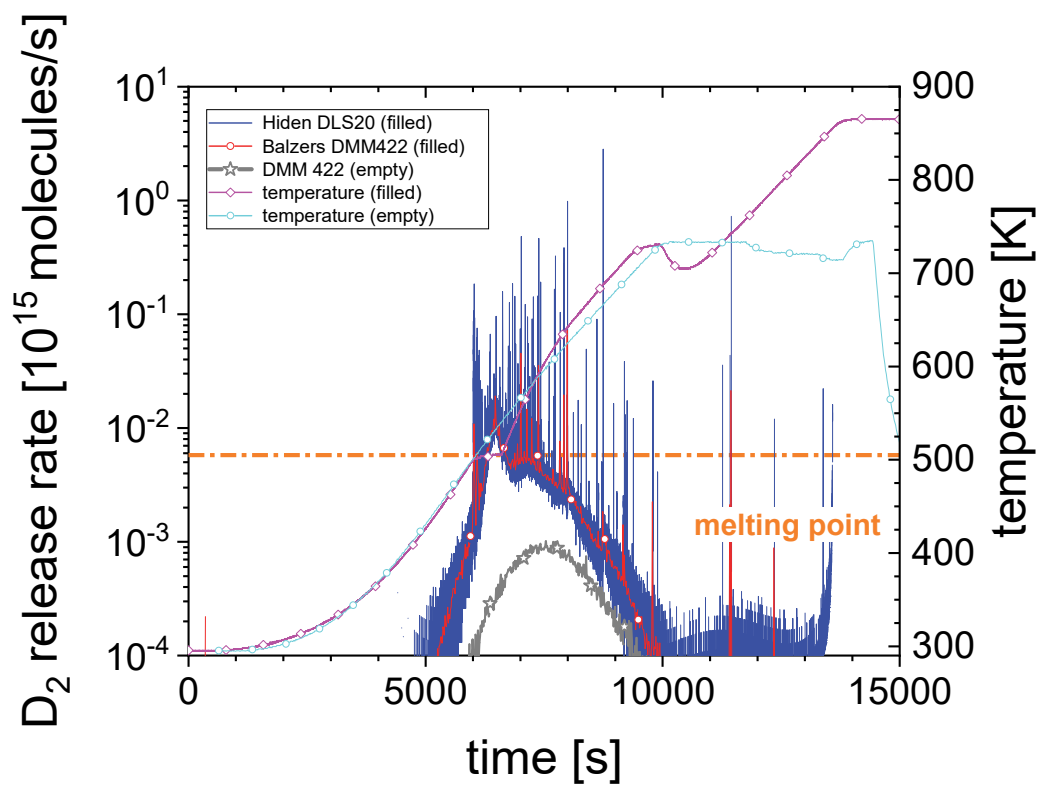

Figure 10: $D_{2}$ release spectra from the sample exposed to $D$ plasma for $1 \mathrm{~h}$ at $515 \mathrm{~K}$, as well as from the empty crucible exposed for $1 \mathrm{~h}$ at $495 \mathrm{~K}$. Due to an unstable sensitivity of the Hiden DLS2O spectrometer, its calibration factor was dynamically adapted to match the Balzers DMM422 in the continuous part of the spectrum. In addition to the continuous release, the Hiden DLS2O accurately captured a large number of $D_{2}$ release spikes, which the Balzers DMM422 mostly missed. In contrast to the samples exposed below the melting point of $S n, D_{2}$ release continues long after the melting plateau. The heating ramp was initially programmed only to $730 \mathrm{~K}$, but then extended to $865 \mathrm{~K}$. The release spectra of the filled and empty crucibles were synchronized such that the temperature of 505 $K$ (corresponding to the melting point) is reached at the same time. Note the absence of the melting plateau for the empty crucible.

At present, the source of the continuous release signal is not fully clear. We can consider one of the following explanations:

1. A very large number of small, overlapping bursts

2. A continuous (mechanical) leakage from the large gas reservoir

3. Permeation of $D_{2}$ (and possibly HD) from the large gas pocket through the $\mathrm{Sn}$ melt

4. A small concentration of $D$ dissolved homogeneously within the Sn melt.

While overlapping bursts can indeed lead to the appearance of a noisy, quasi-continuous release signal for long integration times [28], this hypothesis is discouraged by the fact that the continuous signal appears identical in both mass spectrometers despite the very different integration times. A mechanical leak cannot be excluded, but seems implausible considering the excellent wetting of the $\mathrm{Sn}$ melt on the inner walls of the crucible.

Permeation of $D_{2}$ through the $\mathrm{Sn}$ melt appears quite plausible particularly at higher temperatures. Unfortunately, there is only little and inconsistent data on the solubility of $\mathrm{H}$ isotopes in $\mathrm{Sn}$ (see [3] and references therein), with basically the only agreement being that the heat of solution should be endothermic. Recent modelling effort suggests, however, that the diffusivity of $\mathrm{H}$ isotopes in liquid 
Sn could be very high [29]. It also cannot be excluded that some D permeated through the crucible at high temperatures. In addition, it must be assumed that the alloying of $\mathrm{Sn}$ and $\mathrm{Cu}$ that occurred at high temperatures also had an effect on $D$ release, although it is difficult to predict any details.

Unfortunately, it turned out that during the TDS measurements of the liquid-exposed Sn and the empty crucible, the sensitivity calibration of the Hiden DLS20 was unstable. Based on a comparison of the continuous part of the signal for the Balzers DMM422 and the Hiden DLS20, variations of up to a factor 2 were observed over the time of the TDS ramp and calibration measurement. We therefore used the Balzers DMM422 to cross-calibrate the Hiden DLS20 in the continuous part of the spectrum and interpolated this calibration factor to the spikes in the release spectrum, since the time scale of the sensitivity variations was much slower than that of the spikes. Furthermore, judging by the Hiden DLS20 measurement after subtracting the release from the crucible (i.e., mass 4 only, but the spikes are resolved), about $74 \%$ of the $D$ was released in the continuous part of the spectrum, and only $26 \%$ in the form of spikes.

Nuclear reaction analysis suggested that the concentration of D within the re-solidified Sn exposed at $515 \mathrm{~K}$ should be very low, i.e., of the order of $5 \times 10^{-5}$ at.- $\%$ or less (see section 4$)$. However, assuming a homogeneous $\mathrm{D}$ concentration of this magnitude throughout the entire $2.6 \mathrm{~g}$ of $\mathrm{Sn}$ would lead to a total $D$ content roughly equal to the amount of $D$ released during TDS.

As mentioned before, the $\mathrm{W}$ coating of the $\mathrm{Cu}$ crucible started disintegrating with increasing temperature, and the Sn alloyed with the Cu. After removal from the TDS set-up, the sample appeared not like a re-solidified droplet anymore, but rather like a pile of needle-like structures fused to the crucible with no clear separation. It could, therefore, also be that the reaction of Sn with $\mathrm{Cu}$ influenced the $\mathrm{D}$ release spectrum particularly at higher temperatures.

The data acquired with the Balzers DMM422 spectrometer suggests that in contrast to the pure Sn samples heated without a crucible, a significant part of the total $D$ inventory is not released in the form of $D_{2}$ : A comparable amount of $D$ atoms are desorbed from the filled crucible as HD molecules; for the empty crucible, the HD release is actually substantially larger than the $D_{2}$ release. In terms of raw QMS signals, an even larger amount is released in $\mathrm{HDO}$ and $\mathrm{D}_{2} \mathrm{O}$ molecules (see supplementary S9). Unfortunately, we are presently unable to accurately calibrate the sensitivity of the TESS set-up for water molecules released from the sample, primarily due to possible sticking of these molecules to any vessel walls on the way to the mass spectrometer. Comparing the gas release from the filled and from the empty crucible, it becomes apparent that the $\mathrm{HDO}$ and $\mathrm{D}_{2} \mathrm{O}$ signals can be practically fully explained by release from the crucible. In the case of $H D$, a large part of the desorption spectrum can be attributed to the crucible as well. There are some distinct features, however, that are not present in the crucible spectrum and must therefore originate from the Sn. These are, most notably, the release spikes, but also the low-temperature rising flank and the high-temperature tail of the continuous release spectrum.

\subsection{Comparison to Nuclear Reaction analysis}

The total amounts of $D$ retention within the NRA analysis range of about $20 \mu \mathrm{m}$ and $D$ release measured by TDS are shown in Figure 11. The sources of the statistical and systematic uncertainties of the absolute values from both kinds of measurements were discussed already in sections 2.3 and 2.4. An additional, but not easily quantifiable error arises from the contribution of the crucible to the release of D-containing molecules from the sample exposed at $515 \mathrm{~K}$. While $\mathrm{HDO}$ and $\mathrm{D}_{2} \mathrm{O}$ molecules can most likely be fully attributed to the crucible and is therefore neglected here, the issue of distinguishing $H D$ and $D_{2}$ from $S n$ and crucible remains (see section 5.2). For the samples exposed at 
300 and $495 \mathrm{~K}$, TDS suggests a stronger increase of the D inventory with exposure time than NRA. In addition, while for the $1 \mathrm{~h}$ exposures at these temperatures, NRA and TDS approximately agree, the amounts of $D$ detected by TDS are systematically larger than the amounts of $D$ found by NRA for longer exposure times. This can generally be seen as an indicator for $D$ penetration beyond the NRA analysis range with increasing exposure time. Considering the thick, sponge-like layers formed at 495 $K$, as well as the rather flat $D$ depth profiles, this is rather obvious. $D$ retention within the first $\sim 20 \mu \mathrm{m}$ (dense Sn equivalent) measured by NRA stays approximately constant or even drops slightly at large fluences. At the same time, the steady increase of $D$ release during TDS for increasing plasma exposure time is qualitatively in accordance with the growth of the sponge-like layer. For the samples exposed at $300 \mathrm{~K}$, it is interesting to note that the $D$ retention measured by NRA matches the $D$ release in the main release spike at the melting point for all exposure times, while the total $D$ release exceeds the NRA value. Our interpretation is that the release during melting originates from the disintegration of the subsurface gas bubbles. Their depth (see Figure $3 \mathrm{~b}$ ) coincides with the maximum of the D concentration at the sample surface found by NRA (see Figure 10), which dominates the $D$ retention within the NRA analysis range. Assuming this interpretation is correct, the remaining $D$ release, which is dominated by the low-temperature release peak around $400 \mathrm{~K}$, must originate from $\mathrm{D}$ trapped by defects in the $\mathrm{Sn}$ bulk beyond the NRA range. For the sample exposed at $300 \mathrm{~K}$ for $96 \mathrm{~h}$, the additional D amount in TDS would require a diffusion depth of about $0.4 \mathrm{~mm}$ assuming a bulk D concentration of 0.004 at.-\% as in the far tail of the corresponding NRA depth profile (compare Figure 7). This would correspond to an effective diffusion coefficient of roughly $5 \times 10^{-9} \mathrm{~cm}^{2} \mathrm{~s}^{-1}$. Unfortunately, we have not found any literature data on the diffusion coefficient of $\mathrm{H}$ isotopes in solid Sn. Comparing to other metals, this effective diffusion coefficient is of the same order of magnitude as for $\mathrm{H}$ in $\mathrm{Ni}$, but much slower than, e.g., for $\mathrm{H}$ in $\mathrm{Fe}$ (see, e.g., the review by Fukai and Sugimoto [30]). Figure 11 also highlights clearly that for all Sn samples exposed in the solid state (i.e., at 300 and $495 \mathrm{~K}$ ), D release occurs predominantly in the massive release spike at the melting point, which we assign to disintegration of subsurface bubbles respectively the porous, sponge-like layer upon melting. For liquid $\mathrm{Sn}$ exposed at $515 \mathrm{~K}$, on the other hand, the larger part of the $D$ release occurs continuously, despite the much larger number of spikes in the $D$ release spectrum. 


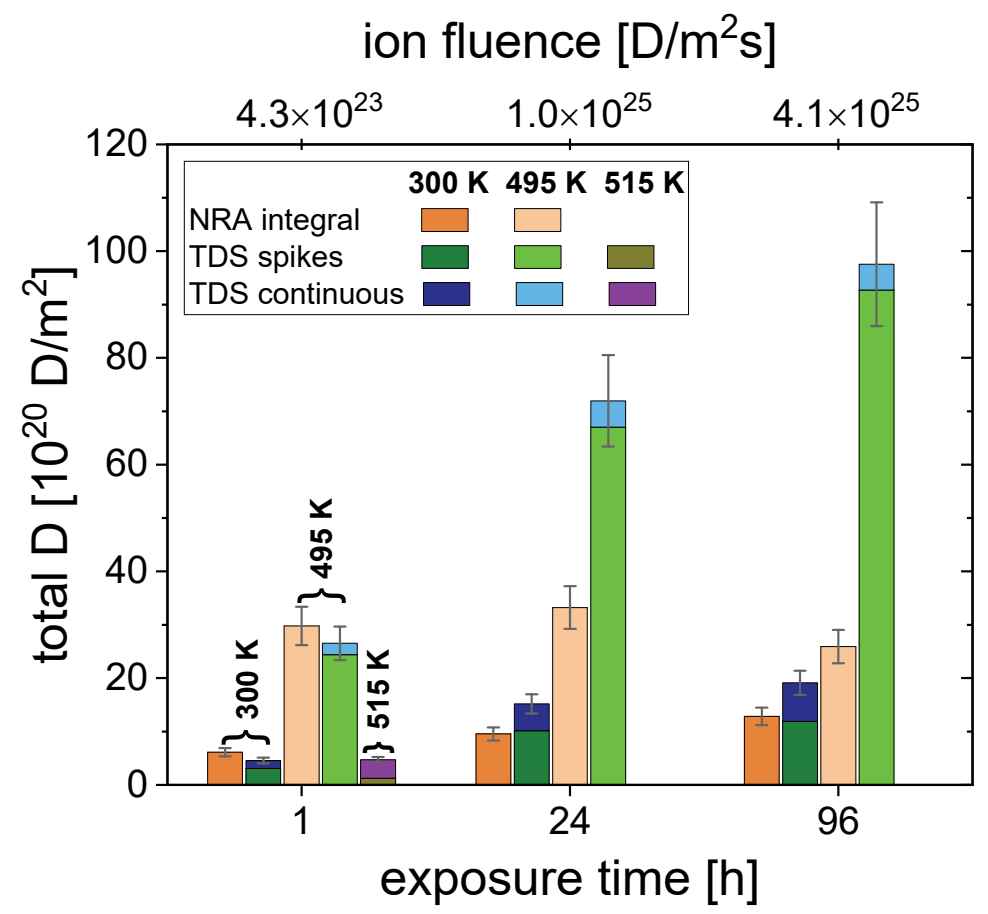

Figure 11: Total amount of $D$ retention (NRA) respectively $D$ release (TDS) of Sn samples exposed to $D$ plasma at the given temperatures. For TDS data, the contribution of $D$ release in spikes and in the continuous part of the spectrum is indicated. For samples exposed at 300 and $495 \mathrm{~K}$, only the release of mass $4\left(D_{2}\right)$ measured by the Hiden DLS20 mass spectrometer needs to be considered. For the sample exposed at $515 \mathrm{~K}$, also a substantial amount of mass 3 (HD) release was found. The total amount of $D$ release was therefore determined by the Balzers DMM422 mass spectrometer. The separation into continuous release and spikes is done based on Hiden DLS2O data. For the $515 \mathrm{~K}$ exposure, the NRA signal was at or below the detection limit. For all signals, the error bars indicated are a combination of statistical and systematic uncertainties. Error bars for TDS signals represent the error of the total $D$ release.

\subsection{Comparison to literature data}

The $D$ retention and release data acquired here can be best compared to the data of Ou et al. [7] acquired for free Sn surfaces. Unfortunately, the particle fluxes used in [7] are not explicitly stated, but a rough estimate based on the stated electron temperature and density in nano-PSI using the Bohm criterion yields values of the same order of magnitude as they were used here. The ratio of ions to radicals is also roughly similar in both experimental set-ups. Comparing our results for solid Sn exposed at $495 \mathrm{~K}$ with the result of [7] at $453 \mathrm{~K}$, in both cases for 1 hour, strikingly similar NRA depth profiles and D release spectra during TDS are found. After exposure of liquid Sn, however, the samples with free Sn surfaces exposed in nano-PSI [7] look rather different than our liquid-exposed samples: While in both works, ejection of Sn droplets was observed, we also observed the accumulation of $D_{2}$ gas in a large cavity at the bottom of the crucible, which caused the liquid $\mathrm{Sn}$ surface to rise to the rim of the crucible within $\sim 1$ hour of exposure (see section 3.2 and Figure 5). On the other hand, the samples containing liquid $S n$ were thickly covered by many small $\mathrm{Sn}$ hemispheres, which points to a very high bubble bursting activity [7]. These droplets typically contained a cavity at the interface between $\mathrm{Sn}$ and crucible wall. We suspect that both effects are closely related, and that the differences may be due to the presence or absence of a magnetic field. 
In $\mathrm{PlaQ}$, the combination of sample current and magnetic field caused the Sn melt to spin (see section 3.2 and video file in the supplementary material). As discussed before, the observed large gas accumulation at the bottom of the crucible cannot be quantitatively explained by diffusion alone. Instead, we propose that particularly the magneto-hydrodynamic forces occurring in PlaQ lead - in combination with the thermal convection - to a considerable convective flow, which can also drag gas bubbles formed in the melt into larger depth. In nano-PSI, there is no magnetic field [7], so only diffusion and thermal convection remain. We therefore propose that bubbles forming in relative proximity to the melt surface do not get dragged away as in PlaQ, but quickly re-surface and burst. This is also in line with the intense spray of Sn droplets observed in [7].

Concerning $\mathrm{D}$ depth profiles in re-solidified, liquid-exposed $\mathrm{Sn}$, Ou et al. report a reduced nearsurface $\mathrm{D}$ concentration compared to solid-exposed $\mathrm{Sn}$, but not nearly as drastically as observed here. This difference may be related to the assumed difference in gas bubble dynamics in the melt (see above). In this work, the $\mathrm{D}_{2}$ bubbles are rather dispersed in the melt (see section 3.2), i.e., on average far beyond the observation range of NRA. This assumption is backed by the observation of numerous small pores throughout the re-solidified $\mathrm{Sn}$ in the metallographic cross-section (see Figure 5 and Supplementary S5). On the other hand, in [7], the bubbles would be rather concentrated near the melt surface, i.e., within the observation range of NRA. Indeed, the amount of D released during TDS is rather similar for our sample exposed at $515 \mathrm{~K}$, and the base part of a sample exposed in nano-PSI at $758 \mathrm{~K}$. This indicates that the observed differences regarding the near-surface $\mathrm{D}$ concentration are indeed rather an issue of $D$ bubble distribution than of total $D$ retention.

Cremona et al. [6] exposed liquid Sn with a free surface at $573 \mathrm{~K}$ in an electron cyclotron resonance plasma source with roughly similar ion fluxes and fluences, but somewhat higher ion energies than in our case. They report a much smaller total $D$ retention than in our experiments or those reported in [7]. In addition, practically all $D$ appears to be located in a thin surface layer of about $100 \mathrm{~nm}$ thickness [6], whereas we, as well as [7], find a substantial, respectively even dominant, quantity of D in large depth by comparing ion beam analysis and TDS. We also note that [6] reports that the majority of the D release occurred via HD molecules. The release of deuterated water molecules, i.e., $\mathrm{HDO}$ and $\mathrm{D}_{2} \mathrm{O}$ was not monitored. Ou et al. [7] have suggested that part of the discrepancy might be due to slow cooling of the samples, as well as due to isotope exchange with $\mathrm{H}$ during storage leading to a further loss of D. Considering that the samples investigated in [6] were stored in air for 2 months before TDS, whereas our samples were stored in vacuum and only removed for analyses, we also consider it plausible that a substantial amount of $D$ was released as deuterated water. At least for tungsten exposed to $D$ plasma, it was already demonstrated that even an exposure to ambient air of only 24 hours can lead to a massive conversion of $D$ to $H D O$ and $D_{2} O$ via reactions of effusing $D$ with surface oxides [31]. In our experiments, the samples were stored in vacuum exsiccators (see sections 2.4 and 2.5) after plasma exposure in order to minimize oxidation. Indeed, release of deuterated water from liquid-exposed $\mathrm{Sn}$ and could be attributed practically fully to the crucible, whereas solid $\mathrm{Sn}$ (without crucibles) showed practically pure $\mathrm{D}_{2}$ release. Similar to our work, Ou et al. have monitored and considered $\mathrm{HDO}$ and $\mathrm{D}_{2} \mathrm{O}$ in the calculation of the total $\mathrm{D}$ inventory [7].

Loureiro et al. have exposed free Sn surfaces to edge plasmas in the limiter tokamak ISTTOK [5]. Two samples were exposed at $\sim 300 \mathrm{~K}$, and two more at $523 \mathrm{~K}$. The ion fluxes were more than 100 times higher in their experiments than in ours, but the exposure time was only 3 seconds per sample, i.e., about 1000 times shorter than even our shortest exposure times. Correspondingly, the incident $D$ ion fluences are substantially lower, and the $D$ transport in $S n$ is also very small due to the short exposure time. In [6] a substantial reduction of $D$ retention comparing solid and liquid $S n$ by a factor of about 3 to 4 was observed. Due to the low $D$ ion fluence, the reported amounts of $D$ are, naturally, also lower than in our experiments and those of Ou et al. [7]. In [5] nuclear reaction analysis of $D$ 
were performed using a fixed ${ }^{3} \mathrm{He}$ energy of $1.2 \mathrm{MeV}$, which probes a depth range of about $2 \mu \mathrm{m}$. As stated above, a very small diffusion of the $D$ beyond the implantation depth is to be expected. Since the maximum of the reaction cross-section for the $D\left({ }^{3} \mathrm{He}, \mathrm{p}\right)^{4} \mathrm{He}$ reaction lies at slightly above 0.6 $\mathrm{MeV}$ [20], i.e., substantially below the Sn surface for these conditions, Loureiro et al. [5] may have systematically underestimated the actual $D$ concentration in the samples exposed th this temperature.

\section{Sn erosion and re-deposition}

\subsection{Sn erosion}

After exposure of solid $\mathrm{Sn}$ at $\mathbf{3 0 0} \mathrm{K}$ and $\mathbf{4 9 5} \mathrm{K}$, we found in good approximation a linear dependence of the weight loss on the plasma exposure time for both cases. Note that the linear fits indicated in Figure 12 both include the origin. This indicates a constant erosion rate, i.e., without any acceleration or suppression of the erosion process by, e.g., surface roughening or chemical modifications of the surface. At $300 \mathrm{~K}$, the average erosion rate was $10.1 \pm 0.1 \mu \mathrm{g} / \mathrm{h}$. At $495 \mathrm{~K}$, the erosion rate was more than 10 times lower at $0.77 \pm 0.05 \mu \mathrm{g} / \mathrm{h}$. Since, as discussed in section 2.2, the ion energy is far below the sputtering threshold for $\mathrm{D}$ on $\mathrm{Sn}$, we attribute this mass loss to chemical erosion by formation of $\mathrm{SnD}_{4}$. The strong temperature dependence of the erosion rate also fits to this conclusion. A similar, plasma-enhanced chemical erosion was already documented by Elg et al. [8]. It is interesting to note that the erosion rate decreases with increasing temperature. However, also for the chemical erosion of carbon and silicon, which are in the same group in the periodic system, chemical erosion in hydrogen isotope plasmas is observed (see, e.g., [32] and references therein). In both cases, the erosion rate rises up to a certain optimal temperature and then decreases again. At an ion energy of $20 \mathrm{eV}$, which is below the sputtering threshold for both $\mathrm{C}$ and $\mathrm{Si}$, this optimal temperature was found to be about $570 \mathrm{~K}$ for $\mathrm{C}$ and about $350 \mathrm{~K}$ for $\mathrm{Si}$, which has the larger atomic number of the two [32]. Extrapolating this trend suggests that the optimal temperature for chemical erosion of Sn should be even lower. Considering also our measurements presented here, we estimate it would be around 300 $\mathrm{K}$, possibly even lower. Particularly for $\mathrm{Sn}$, the decreasing erosion rate at higher temperatures could also be directly related to the instability of stannane gas, $\mathrm{SnD}_{4}$. While due to the endothermic formation of $\mathrm{SnD}_{4}$ [33], the formation rate of $\mathrm{SnD}_{4}$ is expected to increase with temperature, $\mathrm{SnD}_{4}$ decomposition at the hot $\mathrm{Sn}$ surface will also increase. This will compete with $\mathrm{SnD}_{4}$ desorption and thus possibly limit the net erosion rate. In addition, the formation of the thick, sponge-like layer at $495 \mathrm{~K}$ may be an indicator that the absorption of D, maybe also of metastable Sn-D compounds (i.e., stannane or some fragments of it) also competes with $\mathrm{SnD}_{4}$ formation and/or desorption.

In order to calculate the erosion rate of Sn per plasma-exposed area, we need to estimate the active surface of the $\mathrm{Sn}$ samples. Their projected surface of the Sn samples can be derived from the inner diameter of the crucible and is about $0.8 \mathrm{~cm}^{2}$. This surface would be relevant if the impact of plasma particles was the rate-limiting step for the erosion of solid $\mathrm{Sn}$, since their impact rate depends only on the projected surface. The actual curved surface of the re-solidified $S n$ is approximately $1.25 \mathrm{~cm}^{2}$. This surface area would be relevant if the rate-limiting step was $\mathrm{SnD}_{4}$ desorption, or maybe the interaction with the $D_{2}$ background gas. It might be possible to discern these processes by only varying the plasma flux while keeping in particular the $D_{2}$ gas pressure constant. Unfortunately, with the plasma source used here, it is currently not easily possible to vary those parameters independently (see also [9]). Strong roughening of the sample surface (i.e., formation of the spongelike layers shown in Figure 4) should in principle increase the active surface for chemical erosion. On the other hand, it might also lead to a reduced escape probability of $\mathrm{SnD}_{4}$, which would be more likely to decay before leaving the dense network of pores and tendrils. Interestingly, our data 
suggests a roughly constant erosion rate of solid Sn over time, both at $300 \mathrm{~K}$ and $495 \mathrm{~K}$. This could mean either that the processes discussed above are not rate-limiting, or that the effects of increased reaction surface and reduced escape probability of $\mathrm{SnD}_{4}$ coincidentally just compensate under the conditions investigated here. Furthermore, Elg et al. [8] conclude from independent variations of discharge power and gas pressure flanked by plasma modelling that, at least under their conditions, the erosion rate directly depends only on the ion energy flux, and not on the hydrogen radical density. Elg et al. also exclude a direct influence on the neutral gas pressure or flow - they claim that only an indirect effect via the impact of the gas pressure on the ion energy flux is present, and that the local Sn re-deposition rate, which indeed depends on the neutral gas parameters, should be negligible compared to the etching rate. In light of these arguments, we would rather relate the erosion rate to the projected sample surface of $0.8 \mathrm{~cm}^{2}$. At present, we therefore give the estimation that based on the measured erosion rates in $\mu \mathrm{g} / \mathrm{h}$, the erosion rate per plasma-exposed surface area of $\mathrm{Sn}$ is $12.6 \pm 0.2 \mu \mathrm{g} \mathrm{cm} \mathrm{cm}^{-1}$ at $300 \mathrm{~K}$, and $0.97 \pm 0.06 \mu \mathrm{g} \mathrm{cm}^{2} \mathrm{~h}^{-1}$ at $495 \mathrm{~K}$. Please note that the uncertainty estimation does not include the systematic error introduced by the sample surface area. The corresponding erosion yields per $\mathrm{D}$ delivered by ion (in our case, $3 \mathrm{D}$ per $\mathrm{D}_{3}{ }^{+}$ion) are obtained by dividing these values by the atomic mass of $\mathrm{Sn}$ and by the ion flux of $1.2 \times 10^{20} \mathrm{D} / \mathrm{m}^{2} \mathrm{~s}$. Keeping in mind that, compared to the determined erosion rates in $\mu \mathrm{g} / \mathrm{h}$, the exact sample area and ion flux are somewhat uncertain, we tentatively obtain erosion yields of about $1.5 \times 10^{-3} \mathrm{Sn} / \mathrm{D}$ at $300 \mathrm{~K}$, and about $1.1 \times 10^{-4} \mathrm{Sn} / \mathrm{D}$ at $495 \mathrm{~K}$. We would like to stress again that these are not sputter yields, but effective chemical erosion yields that are empirically scaled to the ion flux on the basis of evidence presented by Elg et al. [8]. The detailed nature of this erosion mechanism is presently still unclear. Considering the similarities to carbon erosion by hydrogen isotope plasmas discussed above, it is also plausible that complex, synergistic processes involving both ions and radicals, in analogy to chemical sputtering of carbon [34], may play a role.

\subsection{Sn re-deposition}

On the Mo witness sample, we found a thin layer of about $1.5 \pm 0.1 \times 10^{15} \mathrm{Sn} / \mathrm{cm}^{2}$ of re-deposited $\mathrm{Sn}$ by RBS analysis after exposure to D plasma at $300 \mathrm{~K}$. The layer thickness did not show a clear trend across the Mo sample. It is clear that the $\mathrm{Sn}$ from this layer must originate from chemical erosion of the $\mathrm{Sn}$ in the crucible, i.e., the formation of stannane. However, it is presently not clear whether the deposition occurs via decomposition of $\mathrm{SnD}_{4}$ on the Mo surface, or if the $\mathrm{SnD}_{4}$ already decomposes in the plasma, so that mainly $\mathrm{Sn}$ atoms or ions impinge on the witness sample. It is also interesting to note that net deposition occurs on the Mo sample, while there is net erosion on the $\mathrm{Sn}$ in the crucible, despite the homogeneous temperature across the sample holder and the nearly homogeneous plasma density (see Figure 2). Elucidating the nature and relative weight of the processes involved would be an interesting topic for future plasma and surface chemistry modelling. At $495 \mathrm{~K}$, we could not detect any clear signature of Sn re-deposition on the Mo sample, which is likely due to the much lower net erosion rate at this temperature.

For the two liquid Sn samples exposed at $\mathbf{5 1 5} \boldsymbol{K}$, very high mass losses of $696.6 \pm 1.1 \mu \mathrm{g}$ and $2555.2 \pm 0.8 \mu \mathrm{g}$ were observed after only 60 minutes, respectively 75 minutes, of $D$ plasma exposure at $515 \mathrm{~K}$ (see Figure 12). CLSM as well as SEM (see also supplementary S11) revealed a large amount of $\mathrm{Sn}$ microdroplets. Their number density decreased with increasing distance from the Sn crucible (Figure 14). While the majority of droplets had sizes between less than $1 \mu \mathrm{m}$ and several $\mu \mathrm{m}$, we also found occasional large droplets with sizes of tens of $\mu \mathrm{m}$. The largest documented droplet was about $150 \mu \mathrm{m}$ in diameter. These large droplets were relatively scarce, but occurred everywhere on the Mo witness sample. 3-D surface maps acquired by CLSM as well as SEM images with tilted sample holder 
clearly show that all droplets are very flat. The typical height to diameter ratio, as determined from CLSM surface maps, is of the order of 1:10. This observation means that $\mathrm{Sn}$ also wetted the Mo sample very well under these plasma exposure conditions, just like the main part of the Sn melt wetted the W-coated crucible.

RBS analysis of the Mo witness sample exposed together with liquid Sn at $515 \mathrm{~K}$ for 75 minutes indicated an average amount of re-deposited $\mathrm{Sn}$ of about $17 \times 10^{15} \mathrm{Sn} / \mathrm{cm}^{2}$ closest to the Sn crucible that decayed to $5.2 \times 10^{15} \mathrm{Sn} / \mathrm{cm}^{2}$ at the far edge of the Mo sample (see Figure 13). This is in qualitative agreement with the microscopy observations. However, the presence of the microdroplets means that the amount of Sn detected by RBS is most likely underestimated: While the technique used here is perfectly suitable to detect thin layers of Sn (see section 2.6 and Supplementary S10), at least the larger droplets can be expected to be thick enough that their RBS spectra overlap with the much more intense Mo spectrum. This overlapping part of the Sn spectrum cannot be reliably quantified. As a rough estimate, this will occur for any $\mathrm{Sn}$ droplet thicker than about $80 \mathrm{~nm}$. CLSM 3-D surface maps show that all droplets are relatively flat, with a typical ratio of height to diameter of these droplets of about 1:10. This means that all droplets that are larger than about $1 \mu \mathrm{m}$ will be affected by the spectral overlap in RBS. As a consequence, the amounts of redeposited $\mathrm{Sn}$ discussed here for the exposure at $515 \mathrm{~K}$ must rather be considered a lower limit of the true amount. We expect that the true amount of Sn re-deposited in the form of droplets is substantially higher.

We are convinced that the emission of the observed microdroplets is responsible for the very high mass loss observed for liquid $\mathrm{Sn}$, since extrapolation of the observed temperature dependence of chemical erosion of $\mathrm{Sn}$ would suggest a much lower erosion rate. As already discussed in section 3 , the microdroplets most likely originate from gas bubbles bursting at the surface of the Sn melt.

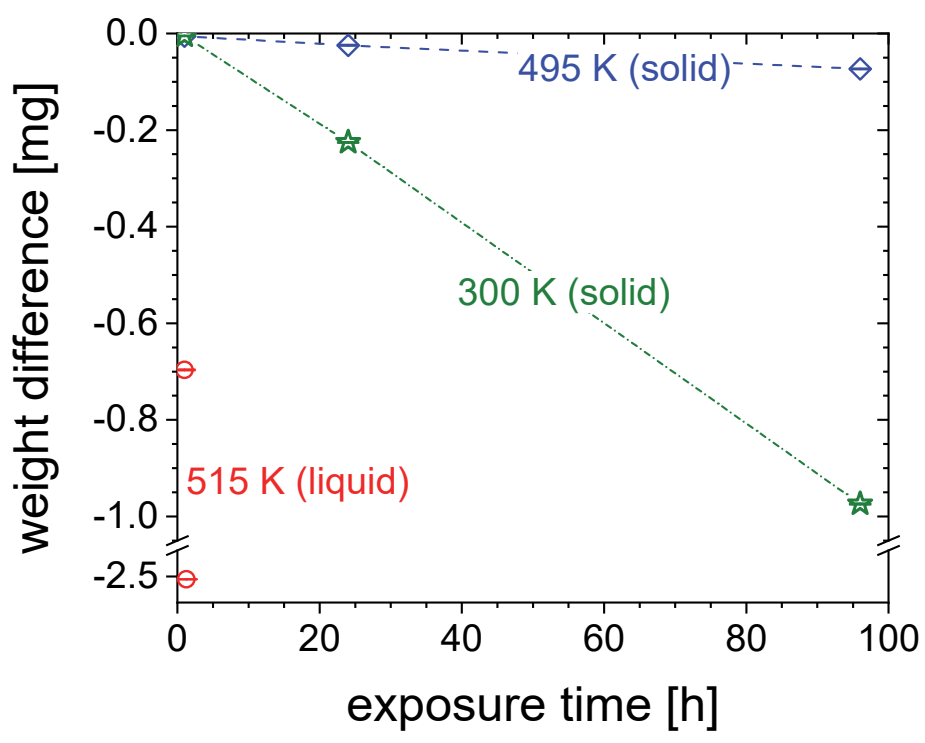

Figure 12: Weight loss measurements of Sn samples exposed to D plasmas at different temperatures for varying exposure times. For solid Sn, net erosion is substantially higher for $300 \mathrm{~K}$ than for $495 \mathrm{~K}$. For each temperature, the erosion rate stays in good approximation constant over 96 hours. Since the ion energy is well below the sputtering threshold, the erosion of solid $S n$ is attributed to chemical erosion by $\mathrm{SnD}_{4}$ formation. For liquid $\mathrm{Sn}$, the erosion rate is much higher, and appears to be predominantly due to the emission of Sn micro-droplets (see also Figures 13 and 14). 


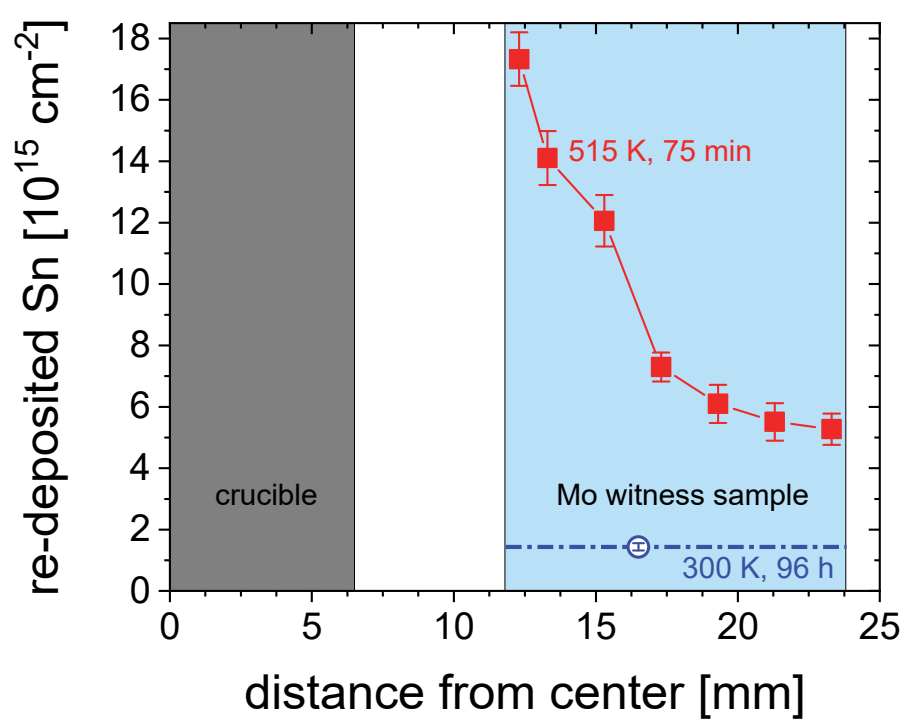

Figure 13: Amount of re-deposited Sn found on Mo witness samples exposed to $D$ plasma together with Sn samples. For exposure of solid Sn at $300 \mathrm{~K}$, a small amount of Sn equivalent to about 1.5 monolayers was found. For exposure of liquid Sn at $515 \mathrm{~K}$, a much higher amount of Sn re-deposition occurred. In this case, a clear increase of re-deposited Sn is found closer to the Sn crucible, which correlates also with the density of Sn micro-droplets visible in optical microscopy (see Figure 14).

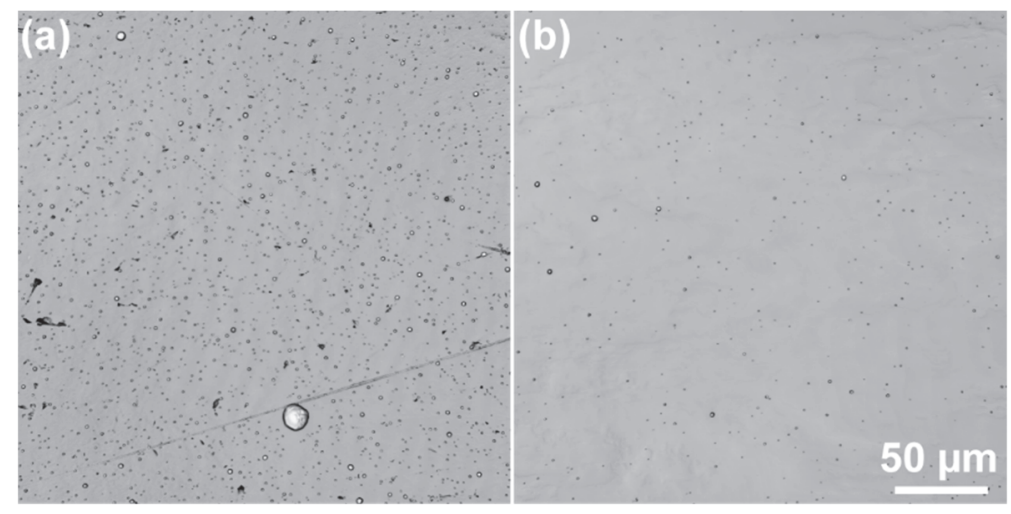

Figure 14: Optical micrographs of Sn micro-droplets on the Mo witness sample exposed together with liquid Sn at $515 \mathrm{~K}$ for $75 \mathrm{~min}$. Panel (a) shows a region close to the $\mathrm{Sn}$ crucible, and panel (b) a region far from the crucible.

One might argue that $\mathrm{Sn}$ is not only re-deposited onto the Mo witness sample, but also onto the crucible, which could distort the weight loss measurements discussed above. To assess the potential error of Sn erosion, we make the following estimate: We first assume that re-deposition should scale with the projected area of the crucible seen by the plasma, and additionally on the entire inner crucible wall that is not covered by the Sn pellet. The Sn pellet is in contact with the crucible wall until about half height, which leaves approximately $0.8 \mathrm{~cm}^{2}$ of the inner crucible surface exposed. The crucible rim and the part of the base not covered by the mounting bracket contribute additionally $0.6 \mathrm{~cm}^{2}$ of projected area, resulting in a total area for re-deposition of $1.4 \mathrm{~cm}^{2}$. We did not find an obvious gradient in the amount of Sn re-deposited onto the Mo witness sample after $96 \mathrm{~h}$ 
plasma exposure at $300 \mathrm{~K}$, and therefore assume that the same amount of $\mathrm{Sn}$ is re-deposited onto the crucible. Generously rounded up, this is about $2 \times 10^{15} \mathrm{Sn} / \mathrm{cm}^{2}$. Considering the molar mass of $\mathrm{Sn}$ of $118.71 \mathrm{~g} / \mathrm{mole}$, the total mass of $\mathrm{Sn}$ that could be deposited onto the crucible under these conditions would then be about $0.6 \mu \mathrm{g}$. This is of the same order as the accuracy of the weighing process, and negligible compared to the total mass loss of about $1 \mathrm{mg}$. For plasma exposure at $495 \mathrm{~K}$, re-deposition onto the Mo sample was too small to be measured. The measured erosion rate was more than 10 times smaller than at $300 \mathrm{~K}$, so we assume that also the re-deposition of $\mathrm{Sn}$ should also be proportionally smaller due to the reduced source of $\mathrm{Sn}$. This would also be consistent with the sensitivity limit of the RBS measurement for Sn re-deposition onto Mo. The relative error of the weight loss due to re-deposition onto the crucible at $495 \mathrm{~K}$ should therefore be negligible as well.

For liquid $\mathrm{Sn}$, it is conceivable that some $\mathrm{Sn}$ droplets should also be deposited onto the rim and outside of the crucible. However, due to the limited accuracy of the RBS measurement and the presently unknown distribution of droplet trajectories, we have no means of estimating this quantity with reasonable accuracy. However, this does not affect our main conclusion that the erosion rate due to droplet ejection from liquid $\mathrm{Sn}$ is much higher than for solid Sn. If anything, we would rather have underestimated the true erosion rate.

\section{Summary and conclusions}

We performed a systematic study of the behavior of solid and liquid tin exposed to a lowtemperature deuterium plasma $\left(1.2 \times 10^{20} \mathrm{D} / \mathrm{m}^{2} \mathrm{~s}\right.$, predominantly $\left.10 \mathrm{eV} / \mathrm{D}\right)$. After exposure at $300 \mathrm{~K}$, the $\mathrm{Sn}$ surface showed a dune-like morphology with many small, partially unstable pores below the surface, which were visible in electron microscopy due to their shallow depth. At $\mathbf{4 9 5} K$, a thick, sponge-like layer containing many open as well as closed pores formed at the Sn surface, which grew in thickness up to several $100 \mu \mathrm{m}$ during 96 hours. The re-solidified surface of Sn exposed in the liquid phase at $\mathbf{5 1 5} \boldsymbol{K}$ showed strong corrugation, in contrast to $\mathrm{Sn}$ molten and re-solidified without plasma exposure. Furthermore, despite the low exposure temperature just above the melting point, the Sn melt had strongly wetted the crucible. In addition, a large gas pocket had formed underneath the melt that pushed the melt upwards. Ou et al also reported that Sn droplets re-deposited onto the crucible walls contained cavities at their interfaces [7]. This may indicate a preference of such gas pockets to precipitate at interfaces between the Sn melt and its substrate.

Weight loss measurements revealed clear indication for chemical erosion - presumably by formation of volatile $\mathrm{SnD}_{4}$ - of solid $\mathrm{Sn}$. The highest erosion rate from solid bulk $\mathrm{Sn}$ of about $10 \mu \mathrm{g} \mathrm{cm}^{-2} \mathrm{~h}^{-1}$ was found at $300 \mathrm{~K}$. After exposure of $\mathrm{Sn}$ for $96 \mathrm{~h}$ at $300 \mathrm{~K}$, a small amount of $\mathrm{Sn}$ re-deposition on the nearby Mo witness sample of about $1.5 \times 10^{15} \mathrm{Sn} / \mathrm{cm}^{2}$ could be identified. At $495 \mathrm{~K}$, the erosion yield is more than 10 times lower. This correlates with the strongly different surface morphologies mentioned above. At $\mathbf{5 1 5} \boldsymbol{K}$, i.e., for $\mathrm{Sn}$ in the liquid state, the dominant mechanism for $\mathrm{Sn}$ mass loss changes from chemical erosion to the emission of microdroplets, most likely caused by bursting of bubbles at the surface of the Sn melt. The droplet emission led to much higher erosion rates of the order of $1 \mathrm{mg} \mathrm{cm}^{-2} \mathrm{~h}^{-1}$. A large amount of droplets was found on the Mo witness sample next to the Sn crucible. Their density decayed with increasing distance from the crucible, i.e., from the droplet source.

D depth profiling using ${ }^{3} \mathrm{He}$-NRA revealed a very sharp and high $\mathrm{D}$ concentration peak of the order of 10 at. $\%$ immediately below the surface for $\mathrm{Sn}$ exposed at $300 \mathrm{~K}$, which had a tail into depth that gradually developed with increasing $D$ ion fluence. The $D$ depth profile acquired from the spongelike, highly porous surfaces formed at $\mathbf{4 9 5} \boldsymbol{K}$ showed a much flatter depth profile with average $D$ 
concentrations of the order of 0.5 at.-\%, particularly at high fluences where the $D$ had penetrated into the Sn much further than the analysis range of about $20 \mu \mathrm{m}$ massive Sn equivalent. These depth profiles also showed an apparent depletion of $D$ right at the surface, which is plausible due to the high porosity of the material. This high porosity also potentially led to increased outgassing of $\mathrm{Sn}$ during cooling after the plasma exposure was finished. For liquid $S n$ exposed at $\mathbf{5 1 5} K$, the D concentration found by NRA was at the detection limit of about $5 \times 10^{-5}$ at.- $\%$.

TDS of Sn samples exposed in the solid state at $300 K$ and $495 K$ yielded almost pure $D_{2}$ release and only minimal amounts of other D-containing molecules such as $\mathrm{HD}, \mathrm{HDO}$ and $\mathrm{D}_{2} \mathrm{O}$. The release occurred mainly in the form of a massive spike during the melting phase of the $\mathrm{Sn}$ samples. The melting phase was recognizable by the temporary stagnation of the sample temperature that is typical for a first-order phase transition. A smaller amount of $D_{2}$ release was also found below the melting point of $\mathrm{Sn}$, particularly after plasma exposure at $300 \mathrm{~K}$, where even a broad secondary lowtemperature peak was observed. After melting, the $D_{2}$ release practically stopped. For $\mathrm{Sn}$ exposed in the liquid phase at $\mathbf{5 1 5} \boldsymbol{K}$, on the other hand, D release started only close to the melting point and continued far past the melting phase. Part of the $D$ was released in a large number of bursts of varying size. Several of these could be clearly attributed to the melt rising to the rim of the crucible, followed by a release of gas from the large cavity underneath the melt. The larger part of $D$ release occurred in the form of a quasi-continuous signal, which most likely originates either from a small, homogeneously dispersed $\mathrm{D}$ concentration in the $\mathrm{Sn}$, or from permeation from the gas pocket through the $\mathrm{Sn}$ melt or through the crucible walls. In addition, other molecules besides $D_{2}$ also contributed strongly to the total $D$ release. Apart from HD molecules, a large fraction of $D$ was released as deuterated water, although this amount could not be reliably quantified due to the very difficult calibration of the TDS set-up for water molecules. Since the Sn had started to strongly wet the $\mathrm{W}$ surface shortly after the plasma exposure started, it could not be removed from the crucible anymore, i.e., it had to be degassed while still inside the crucible. Comparison with the release from the emptied crucible exposed at $495 \mathrm{~K}$ for $1 \mathrm{~h}$ shows that practically all deuterated water came from the crucible, as well as the majority of the HD molecules.

Compared to tungsten as a solid candidate material for fusion reactor plasma-facing components, the amount of $\mathrm{D}$ retained particularly in solid $\mathrm{Sn}$ at $495 \mathrm{~K}$ is considerable. E.g., tungsten pre-irradiated by $20 \mathrm{MeV} \mathrm{W}^{6+}$ ions in order to simulate neutron damage can locally retain comparable $\mathrm{D}$ concentrations at 500-600 $\mathrm{K}$ and similar ion flux and energy [35]. Liquid Sn, at least in our experiments, shows much lower local $D$ concentration, but the observed efficient transport of $D$ into depth - resulting in the quick growth of the gas pocket here - could still lead to quickly rising $D$ retention throughout the bulk of the liquid $\mathrm{Sn}$, or in precipitating gas pockets. In addition, results by Ou et al [7] indicate that liquid Sn inside a molybdenum CPS exposed to high-flux D plasma (i.e., in a situation closer to reactor conditions) could exhibit significantly higher $D$ retention than observed in our experiments. In addition, the design and material of a CPS could have substantial influence on the $D$ retention in a fusion reactor component [7].

Overall, we observe a strong, correlated variation of the behavior of Sn exposed to D plasma at different temperatures for the aspects of $S n$ erosion, $D$ retention and release as well as $S n$ morphology evolution. We speculate that this is strongly tied to the chemistry of $\mathrm{Sn}$ and $\mathrm{H}$ isotopes, i.e., the formation and decay of volatile, metastable stannane molecules. We tentatively propose that there are competing processes of $\mathrm{SnD}_{4}$ formation, desorption, decay and maybe also to dissolution of $D$ atoms or Sn-D compounds in the Sn. Unfortunately, we have not found any data on the possible solubility of, e.g., $\mathrm{SnD}_{4}$ in $\mathrm{Sn}$. The interplay of these processes could plausibly explain the apparently massive formation of gas bubbles far deeper than the implantation range of $D$ ions. This, in turn, appears to be a crucial ingredient in the formation of the sponge-like layer at $495 \mathrm{~K}$, or of the 
large gas pocket at $515 \mathrm{~K}$. If stannane, or other metastable Sn-D compounds, would readily dissolve and diffuse in Sn, it could easily transport D deep into the Sn. Decomposition of such compounds with a certain time delay would then lead to the formation of $D_{2}$-filled bubbles in large depths. In the case of exposure at $495 \mathrm{~K}$, this would result in the formation of the sponge-like layer. For exposure in the liquid state, this would lead to bubbles that are created sufficiently far below the surface so that they do not immediately burst, but can be dragged along by convective flows in the Sn melt.

Unfortunately, the details of the interaction of $\mathrm{H}$ isotopes, $\mathrm{Sn}$ and stannane in a plasma environment are, to our best knowledge, largely unknown. We therefore plan to investigate the role of Sn-D chemistry for the observed effects in more detail in the near future. To accomplish this, we plan to expose Sn samples to He plasmas under similar conditions as used here for D plasma exposure. For example, the sponge-like layer on Sn exposed to D plasma at $495 \mathrm{~K}$ reminds of tungsten nano-fuzz created by high-flux He plasma irradiation of tungsten at high temperatures [36], although the structures observed in our work are much coarser. It remains to be seen whether He plasma irradiation can create structures similar to the sponge-like layer observed here, or rather to tungsten nano-fuzz, or even no related structures at all.

Regarding the potential use of liquid $\mathrm{Sn}$ in the divertor region of a future fusion reactor, our study reveals several effects that could potentially lead to operational risks and therefore need to be investigated further. Sn would be liquid at the divertor, where the highest heat fluxes to the plasmafacing components occur. Here, we would predict only moderate $\mathrm{H}$ isotope retention. The expected strong MHD flows in the melt due to large currents and strong magnetic fields, or maybe even due to intentional forced convection of the liquid metal [27], may increase overall $\mathrm{H}$ isotope uptake compared to our study. This might be desired in the light of controlling recycling, but at the price that more attention has to be paid to efficiently reclaiming tritium from the liquid metal in order to maintain tritium self-sufficiency and ensure nuclear safety. We also predict strongly reduced chemical erosion compared to $300 \mathrm{~K}$. On the other hand, Sn erosion by microdroplet emission caused by bursting gas bubbles could be substantial. It is likely that these droplets would eventually come to rest in cooler regions, where the Sn could be in its solid phase. Here, for example, the observed sponge-like layers could form, which, according to our results, can potentially store substantially larger amounts of $\mathrm{H}$ isotopes than the liquid $\mathrm{Sn}$. Indeed, Balden et al. have observed various deposits of $\mathrm{Sn}$ on a sample consisting of several W monoblocks brazed to a cooling tube that was exposed to very high fluences of $D$ and He plasmas in Magnum-PSI [37]. The deposits even occurred deep inside the gaps between the $\mathrm{W}$ blocks. Sn droplets were found as well as the sponge-like structures reported here, and some Sn-containing mixed material deposits. It is important to note that the $\mathrm{Sn}$ deposits did not originate from $\mathrm{Sn}$ intentionally exposed to the plasma together with the $\mathrm{W}$ monoblocks, but from a previous experimental campaign investigating liquid metals (see [2]). This highlights a high tendency of Sn re-deposits to migrate around a device. The relatively high chemical erosion rate at $300 \mathrm{~K}$ might appear as a possible approach to remove such $\mathrm{Sn}$ deposits. A similar approach was suggested by Elg et al., albeit in a different context [8]. On the other hand, it is well known from fusion experiments with carbon walls that chemical erosion leads to re-deposition of eroded carbon in remote areas. There, it is challenging to remove, or even to accurately keep track of, and can potentially lead to large amounts of tritium retention due to co-deposition (see, e.g., [38, 39]). Also, as shown by Ou et al., Sn droplets can travel a substantial distance even against a highintensity plasma beam [7], which might be an issue for fusion reactor plasma operation. Another point is the observation of the large gas pocked forming below the $\mathrm{Sn}$ melt in our experiment within a rather short time. Such a gas pocket could potentially interfere with the $\mathrm{Sn}$ flow in a liquid divertor concept, or reduce the contact to a heat sink at the far side of the component. Since in our experiment, heat and particle fluxes, electrical currents and magnetic fields were all much weaker than it would be expected for a fusion reactor, it is likely that convection driven by thermal gradients 
and magneto-hydrodynamics would be substantially stronger for a divertor component. Since we argued that the rapid gas pocket formation cannot be explained by solute $\mathrm{H}$ isotope diffusion in $\mathrm{Sn}$, but rather by convective flows, this might also be substantially accelerated. It remains to be seen if the effects observed here, particularly those related to gas bubble formation, only occur in a freely convecting $\mathrm{Sn}$ melt, like in our experiment, or also, e.g., in a capillary porous system for $\mathrm{Sn}$ retention. For example, Ou et al. also observed massive $\mathrm{Sn}$ droplet emission from liquid $\mathrm{Sn}$ in a CPS retainer exposed to a high-flux D plasma [7]. In an upcoming experimental campaign, it is planned to assess how a CPS could mitigate the adverse effects of droplet emission and gas pocket formation, or if instead the correct functioning of a CPS could be hampered, e.g., by gas pockets blocking re-supply of Sn from a reservoir to the component surface. While the alternative concept of a fast-flowing liquid metal divertor (i.e., with forced convection) might suppress droplet ejection by bursting bubbles, it would also promote the convective transport of D away from the plasma-exposed surface [27]. This could foster the formation of gas pockets, e.g., in the liquid Sn supply lines or reservoirs. Based on our fundamental research presented here, we therefore see an urgent need for further experimental and theoretical studies of the chemistry of $\mathrm{Sn}$ in hydrogen isotope plasmas, as well as the dynamics of gas bubble formation and transport for any proposed liquid Sn divertor concept.

Finally, the plasma-assisted wetting observed during our experiments with liquid Sn might be beneficial for the fabrication of liquid-metal plasma-facing components with capillary porous systems, since it would, e.g., allow infiltration of a tungsten CPS with Sn at low temperatures.

\section{Acknowledgement}

This work has been carried out within the framework of the EUROfusion Consortium and has received funding from the Euratom research and training programme 2014-2018 and 2019-2020 under grant agreement No 633053. The views and opinions expressed herein do not necessarily reflect those of the European Commission.

\section{A. Appendix: Flux balance model for permeation}

Let us assume that for a liquid Sn sample in steady state, diffusion-limited boundary conditions occur on both the plasma-exposed surface and at the opposite surface, i.e., at the interface to the crucible, respectively to a gas-filled cavity. "Diffusion-limited" means that recombination and desorption of D at the surface is sufficiently fast so that the rate-limiting step for the actual desorption rate is the diffusive flux of $D$ to the respective surface. These boundary conditions are equivalent to the solute $D$ concentration being zero at either surface.

In this case, the flux of implanted particles, $\Gamma_{\text {impl, }}$ is exactly balanced by the sum of the re-emission flux of particles diffusing back to the exposed surface and desorbing there, $\Gamma_{r e}$, and the flux of particles permeating through the sample, $\Gamma_{\text {perm }}$. In steady state, the solution of Fick's laws also yields linear concentration gradients from the implantation range, where the concentration is maximal, to either surface, where the concentration is zero. According to Fick's law, the diffusion flux is proportional to the concentration gradient times the diffusion coefficient. From that, one can already see that for typical plasma exposure conditions (see also below), where the implantation range $r_{i m p l}$ is small compared to the sample thickness $d, \Gamma_{\text {perm }}$ is much smaller than $\Gamma_{r e}$. Consequently,

$$
\Gamma_{\text {impl }}=\Gamma_{\text {re }}+\Gamma_{\text {perm }} \approx \Gamma_{\text {re }}
$$


The concentration at the implantation range, $c_{\max }$, can therefore with good accuracy be estimated from

$$
\Gamma_{i m p l}=\frac{c_{\max }}{r_{\text {impl }}} \cdot D
$$

where $D$ is the diffusion coefficient of deuterium in the sample. For calculating $\Gamma_{\text {perm }}$, one simply takes the same $c_{\max }$ and $\mathrm{D}$, and replaces $r_{\text {impl }}$ by $\left(d-r_{\text {impl }}\right)$. Calculating finally the ratio of $\Gamma_{\text {perm }}$ and $\Gamma_{\text {impl, }}$ $c_{\max }$ and $D$ cancel out, and $r_{\text {impl }}$ can be neglected in the denominator $\left(d-r_{\text {impl }}\right)$ :

$$
\frac{\Gamma_{p e r m}}{\Gamma_{\text {impl }}}=\frac{r_{i m p l}}{d-r_{\text {impl }}} \approx \frac{r_{i m p l}}{d}
$$

To summarize: If the dominant process of $D$ transport in the $S n$ melt was diffusion, the fraction of the implanted particle flux that permeates through the melt into the gas filled cavity at the bottom of the crucible is in good approximation proportional to the ratio of the implantation range and the sample thickness, and it is independent of the sample temperature or the exact value of the diffusion coefficient. The only prerequisite are the diffusion-limited boundary conditions mentioned above, and that particle transport occurs by random walk, i.e., directed particle fluxes are negligible.

This model can of course also be applied to other kinds of samples, given that the same diffusionlimited boundary conditions apply ("DD" case). Along with the other extreme case of recombinationlimited boundary conditions on both surfaces ("RR" case), as well as mixed cases ("RD" or "DR" cases), it is well-known in the context of ion- or plasma-driven permeation and discussed in great detail, e.g., in [26].

\section{References}

[1] J.W. Coenen et al, Phys. Scr. T159 (2014) 014037

DOI : 10.1088/0031-8949/2014/T159/014037

[2] T.W. Morgan et al, Plasma Phys. Control. Fusion 60 (2018) 014025

DOI: 10.1088/1361-6587/aa86cd

[3] P.W. Humrickhouse, IEEE Trans. Plasma Sci. 47(7) (2019) 3374-3379 DOI: 10.1109/TPS.2019.2919034

[4] M. Poradziński et al, Fusion Eng. Des. 124 (2017) 248-251 DOI : 10.1016/j.fusengdes.2017.04.131

[5] J.P.S. Loureiro et al, Nucl. Mater. Energy 12 (2017) 709-713 DOI: dx.doi.org/10.1016/j.nme.2016.12.026

[6] A. Cremona et al, Nucl. Mater. Energy 17 (2018) 253-258 DOI: 10.1016/j.nme.2018.11.010

[7] W. Ou et al, Nucl. Fusion 60 (2020) 026008 DOI: 10.1088/1741-4326/ab5cd4

[8] D.T. Elg et al, Plasma Chem. Plasma Process. 38 (2018) 223-245 DOI: $10.1007 / \mathrm{s} 11090-017-9852-4$

[9] A. Vertkov et al, Fusion Eng. Des. 117 (2017) 130-134 DOI : 10.1016/j.fusengdes.2017.01.041

[10] A. Manhard et al, Plasma Sources Sci. Technol. 20 (2011) 015010 DOI: 10.1088/0963-0252/20/1/015010 
[11] S. Kapser et al, Nucl. Mater. Energy 12 (2017) 703-708

DOI : 10.1016/j.nme.2016.11.019

[12] A. Manhard et al, Nucl. Fusion 57 (2017) 126012

DOI: 10.1088/1741-4326/aa82c8

[13] A. Mutzke et al, (2019) SDTrimSP Version 6.00 (IPP 2019-2)

Garching: Max-Planck-Institut für Plasmaphysik

DOI: $10.17617 / 2.3026474$

[14] W. Eckstein (2009), Reflection (Backscattering) (IPP 17/12), Garching: Max-Planck-Institut für Plasmaphysik; http://hdl.handle.net/11858/00-001M-0000-0026-F340-E

[15] W. Eckstein (2010), Penetration (Range) (IPP 17/20), Garching: Max-Planck-Institut für Plasmaphysik; http://hdl.handle.net/11858/00-001M-0000-0026-F049-1

[16] P.N. Maya, J. Nucl. Mater. 480 (2016) 411-419

DOI: 10.1016/j.jnucmat.2016.08.007

[17] Z. Yang et al., Fusion Eng. Des. 85 (2010) 1517-1520

DOI: 10.1016/j.fusengdes.2010.04.018

[18] X. Yang, A. Hassanein, J. Nucl. Mater. 434 (2013) 1-6

DOI: 10.1016/j.jnucmat.2012.10.045

[19] S. V. Stankus, R. A. Khairulin, High Temp 44 (2006) 389-395

DOI: 10.1007/s10740-006-0048-5

[20] B. Wielunska et al, Nucl. Instr. Meth. B 371 (2016) 41-45

DOI: 10.1016/j.nimb.2015.09.049

[21] K. Schmid, U. von Toussaint, Nucl. Instr. Meth. B 281 (2012) 64-71

DOI: 10.1016/j.nimb.2012.03.024

[22] M. Mayer, SIMNRA User's Guide, Tech. rep. IPP 9/113

Garching: Max-Planck-Institut für Plasmaphysik, 1997

[23] M. Mayer, Nucl. Instr. Meth. B 332 (2014), 176-180

DOI: 10.1016/j.nimb.2014.02.056

[24] J.F. Ziegler, SRIM-2013, http://www.srim.org

[25] E. Salançon et al, J. Nucl. Mater. 376(2) (2008) 160-168

DOI: 10.1016/j.jnucmat.2008.02.070

[26] T. Tanabe et al, J. Nucl. Mater. 145-147 (1987) 305-308

DOI: 10.1016/0022-3115(87)90349-7

[27] Y. Hirooka et al. Fusion Engineering and Design 117 (2017) 140-144

DOI: 10.1016/j.fusengdes.2016.06.028

[28] A. Manhard et al, Phys. Scr. T145 (2011) 014038

DOI: 10.1088/0031-8949/2011/T145/014038

[29] X. Liu et al, J. Chem. Phys. 147 (2017) 064505

DOI: $10.1063 / 1.4997635$

[30] Y. Fukai, H. Sugimoto, Advances in Physics 34(2) (1985) 263-326

DOI: 10.1080/00018738500101751

[31] K. Moshkunov et al., J. Nucl. Mater. 404 (2010) 174-177

DOI: 10.1016/j.jnucmat.2010.07.011

[32] M. Balden, J. Roth, J. Nucl. Mater. 279(2-3) (2000) 351-355

DOI: 10.1016/S0022-3115(00)00032-5

[33] H. Gamsjäger, T. Gajda, J. Sangster, S.K. Saxena, W. Voigt: "Chemical Thermodynamics of Tin", ISBN 978-92-64-99206-1, pp. 135-136, (C) OECD 2012

[34] W. Jacob and J. Roth, Chemical Sputtering, in: Sputtering by Particle Bombardment IV by R. Behrisch and W. Eckstein (Eds.), pp. 329-400, Springer Verlag, Berlin 2007 
[35] O.V.Ogorodnikova, K.Sugiyama, J. Nucl. Mater. 442(1-3) (2013) 518-527 DOI: 10.1016/j.jnucmat.2013.07.024

[36] M.J. Baldwin and R.P. Doerner Nucl. Fusion 48 (2008) 035001

DOI: 10.1088/0029-5515/48/3/035001

[37] M. Balden et al., Phys. Scr. T171 (2020) 014026

DOI: $10.1088 / 1402-4896 / a b 4479$

[38] M. Mayer et al., Phys. Scr. T111 (2004) 55-61

DOI: 10.1238/Physica.Topical.111a00055

[39] J. Roth et al., J. Nucl. Mater. 390-391 (2009) 1-9

DOI: 10.10106/j.jnucmat.2009.01.037 


\title{
Deuterium retention in solid and liquid tin after low-temperature plasma exposure
}

\author{
A. Manhard, T. Schwarz-Selinger, M. Balden, T. Dürbeck, H. Maier, R. Neu
}

Max Planck Institute for Plasma Physics, Boltzmannstr. 2, 85748 Garching, Germany

\section{Supplementary material and index of video and animation files}

This supplementary shows some additional data for the interested reader and provides additional information to the main article.

Figure $\mathbf{S 1}$ shows a representative Langmuir probe characteristic that was used to estimate the plasma potential in the $\mathrm{D}$ plasma discharges.

Figures $\mathbf{S} \mathbf{2}$ and $\mathbf{S} 3$ depict simulation results of the $\mathrm{D}$ implantation profile and the energy-dependent physical sputter yield of D on Sn using the binary collision approximation code SDTrimSP 6.0 [13]. Table $\mathbf{S 4}$ lists the data underlying Figure S3.

Figures S5-S7 show high-resolution overview images as well as detail images of the metallographic cross-section prepared from re-solidified $\mathrm{Sn}$ exposed to plasma in the liquid state at $515 \mathrm{~K}$ for 75 minutes.

Table $\mathbf{S} 8$ lists the time delays between plasma exposure and NRA as well as TDS analysis for all samples discussed in this work.

Figure $\mathbf{S 9}$ compares the emission of typical D-containing molecules $\left(\mathrm{HD}, \mathrm{D}_{2}, \mathrm{HDO}\right.$ and $\left.\mathrm{D}_{2} \mathrm{O}\right)$ during thermal desorption spectroscopy (TDS) from the Sn sample exposed at $515 \mathrm{~K}$ for 60 minutes together with its crucible, and the emptied crucible that was exposed at $495 \mathrm{~K}$ for 60 minutes.

Figure S10 illustrates the sensitivity of Rutherford Backscattering Spectroscopy (RBS) for very thin layers of $\mathrm{Sn}$ re-deposited onto a Mo witness sample.

Figures S11 and S12 show an SEM image of Sn microdroplets on an Mo witness sample exposed together with $\mathrm{Sn}$ at $515 \mathrm{~K}$, as well an element distribution map acquired by energy-dispersive X-ray analysis (EDX) and the corresponding X-ray sum spectrum.

In addition, an index of supplementary multimedia files (in-situ videos and animated sequences of SEM images) including detailed explanations is provided. The multimedia files have been reduced in size and compressed for easier accessibility.

Files named $\mathbf{S n} \mathbf{5} \mathbf{5 1 5} \boldsymbol{K}^{*} . \mathbf{m p 4}$ show in-situ videos acquired by optical and infrared cameras of $\mathrm{Sn}$ exposed to plasma in the liquid state.

Files named Sn_495K_D-plasma-exposure*.mp4 show in-situ videos acquired by optical camera of $\mathrm{Sn}$ exposed to D plasma just below the melting point. Corresponding files with slicing and tilting in their file names are animations built from SEM image of FIB cross-sections of these samples.

Files named $S n_{3} 300 K^{*} . m p 4$ show a sequence of in-situ images acquired just before and after plasma exposure, as well as SEM image sequences illustrating the instability of superficial pores in these samples.

Raw data as well as high-resolution image sequences and videos are available on request. Contact: Dr. Armin Manhard (armin.manhard@ipp.mpg.de) 


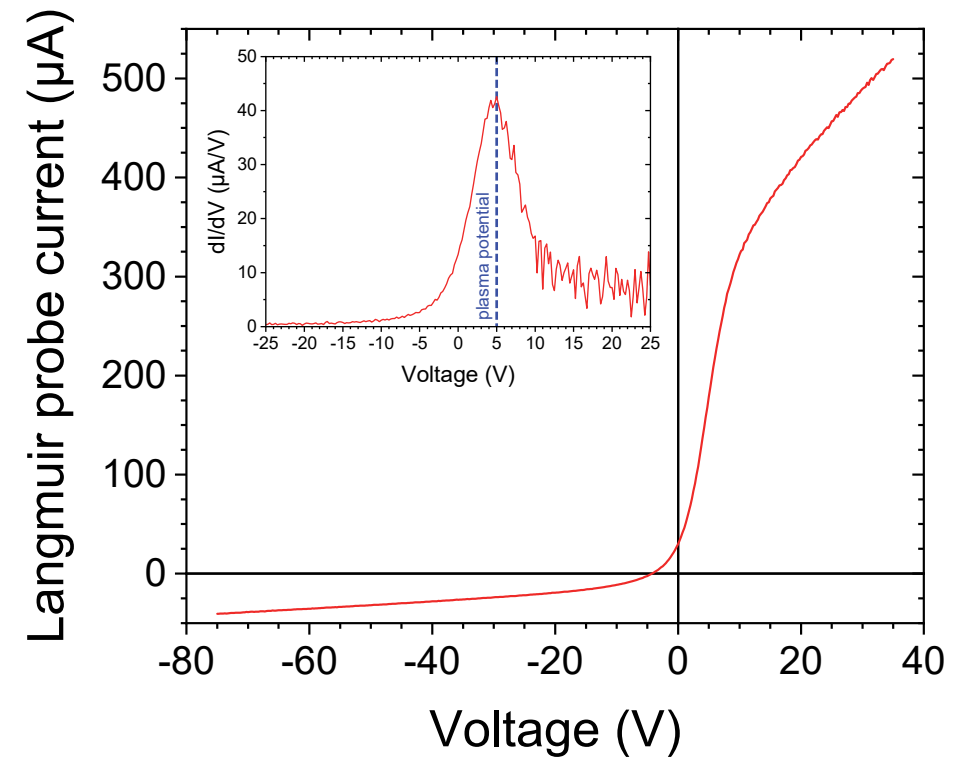

Figure S1: Langmuir probe characteristic and first derivative (inset) acquired during D plasma exposure of a Sn sample. The data was acquired at the location of the Sn sample (Position $x=83 \mathrm{~mm}$, see Figure 2 in the main article). The plasma potential is about $5 \mathrm{~V}$. Together with the sample bias of $-25 \mathrm{~V}$, this leads to an impact energy of $30 \mathrm{eV} /$ ion, respectively $10 \mathrm{eV} / \mathrm{D}$ considering $\mathrm{D}_{3}{ }^{+}$ions.

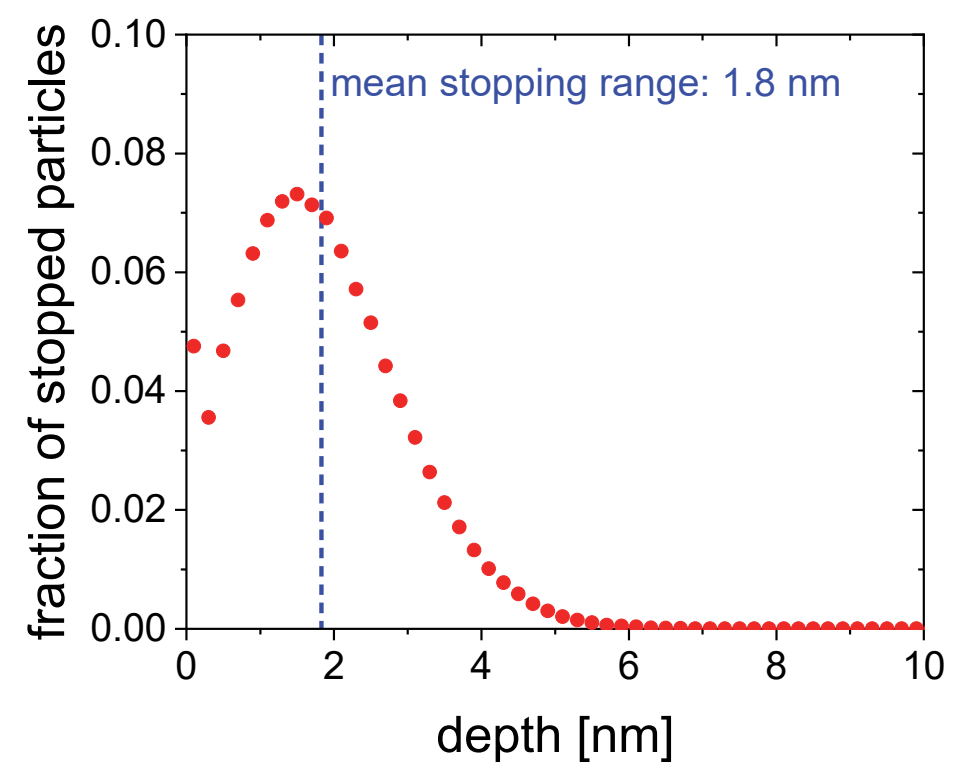

Figure S2: Implantation profile of D into Sn considering D ions with a kinetic energy of $10 \mathrm{eV} / \mathrm{D}$ (i.e., $\mathrm{D}_{3}{ }^{+}$ions with an energy of $30 \mathrm{eV}$, see Figure $\mathrm{S} 1$ ), calculated using the binary collision approximation (BCA) code SDTrimSP 6.0 [13]. $10^{6}$ projectiles were used for the simulation. The resulting mean stopping range is about $1.8 \mathrm{~nm}$, the fraction of backscattered projectiles is about $65 \%$. The calculations are based on a hypothetical amorphous target material and do not take into account any effects due to crystallinity, such as ion channeling. 


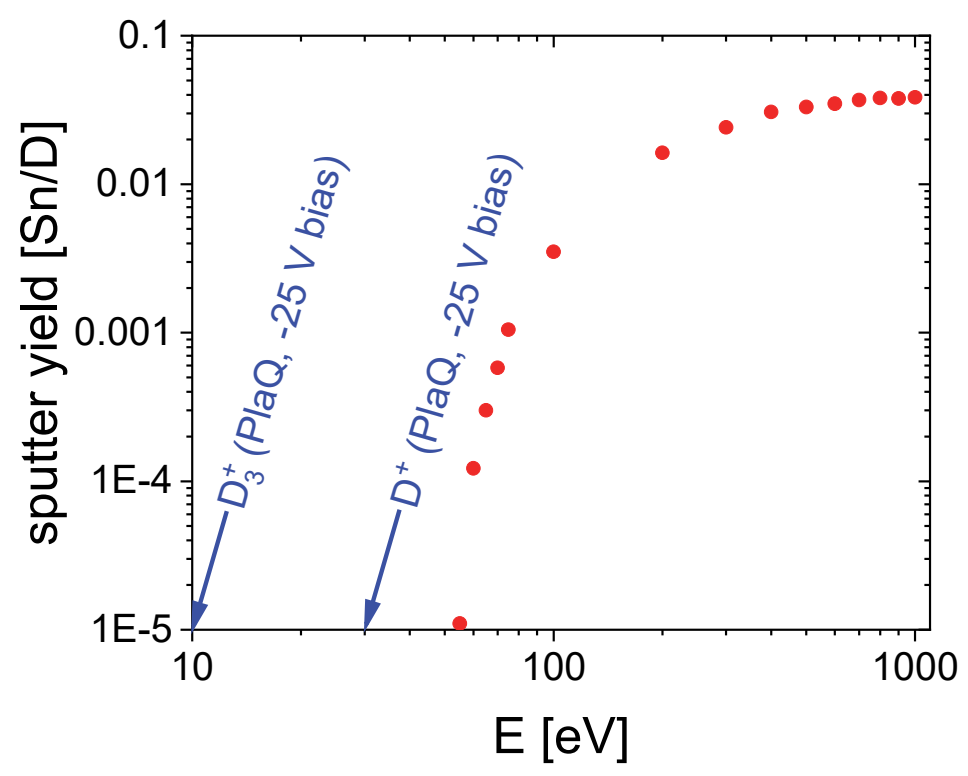

Figure S3: Sputter yield of D impacting on Sn, calculated using SDTrimSP 6.0. The two arrows indicate the kinetic energy per deuteron for the dominant $\mathrm{D}_{3}{ }^{+}$ion species, as well as for the minority species $\mathrm{D}^{+}$, which delivers the highest-energy deuterons in the plasmas used here. Please note that these sputter yields do not consider any chemical effects or temperature-enhanced sputtering (for examples of such effects, see, e.g., Refs. $[2,8]$ in the main article).

Table S4: Table of simulation results for the sputter yield of $\mathrm{D}$ impinging on $\mathrm{Sn}$, calculated by SDTrimSP 6.0.

\begin{tabular}{|l|l|l|}
\hline \multicolumn{1}{|c|}{$\begin{array}{c}\text { impact energy } \\
(\mathrm{eV})\end{array}$} & \multicolumn{1}{|c|}{ number of projectiles } & \multicolumn{1}{c|}{$\begin{array}{c}\text { sputter yield } \\
\text { (Sn/D) }\end{array}$} \\
\hline 50 & 1000000 & 0 \\
\hline 55 & 1000000 & $1.10 \mathrm{E}-05$ \\
\hline 60 & 1000000 & $1.22 \mathrm{E}-04$ \\
\hline 65 & 1000000 & $3.00 \mathrm{E}-04$ \\
\hline 70 & 1000000 & $5.81 \mathrm{E}-04$ \\
\hline 75 & 100000 & 0.00105 \\
\hline 100 & 100000 & 0.00351 \\
\hline 200 & 100000 & 0.01631 \\
\hline 300 & 100000 & 0.02418 \\
\hline 400 & 100000 & 0.03073 \\
\hline 500 & 100000 & 0.03309 \\
\hline 600 & 100000 & 0.03492 \\
\hline 700 & 100000 & 0.03693 \\
\hline 800 & 100000 & 0.03809 \\
\hline 900 & 100000 & 0.03784 \\
\hline 1000 & 100000 & 0.03847 \\
\hline
\end{tabular}



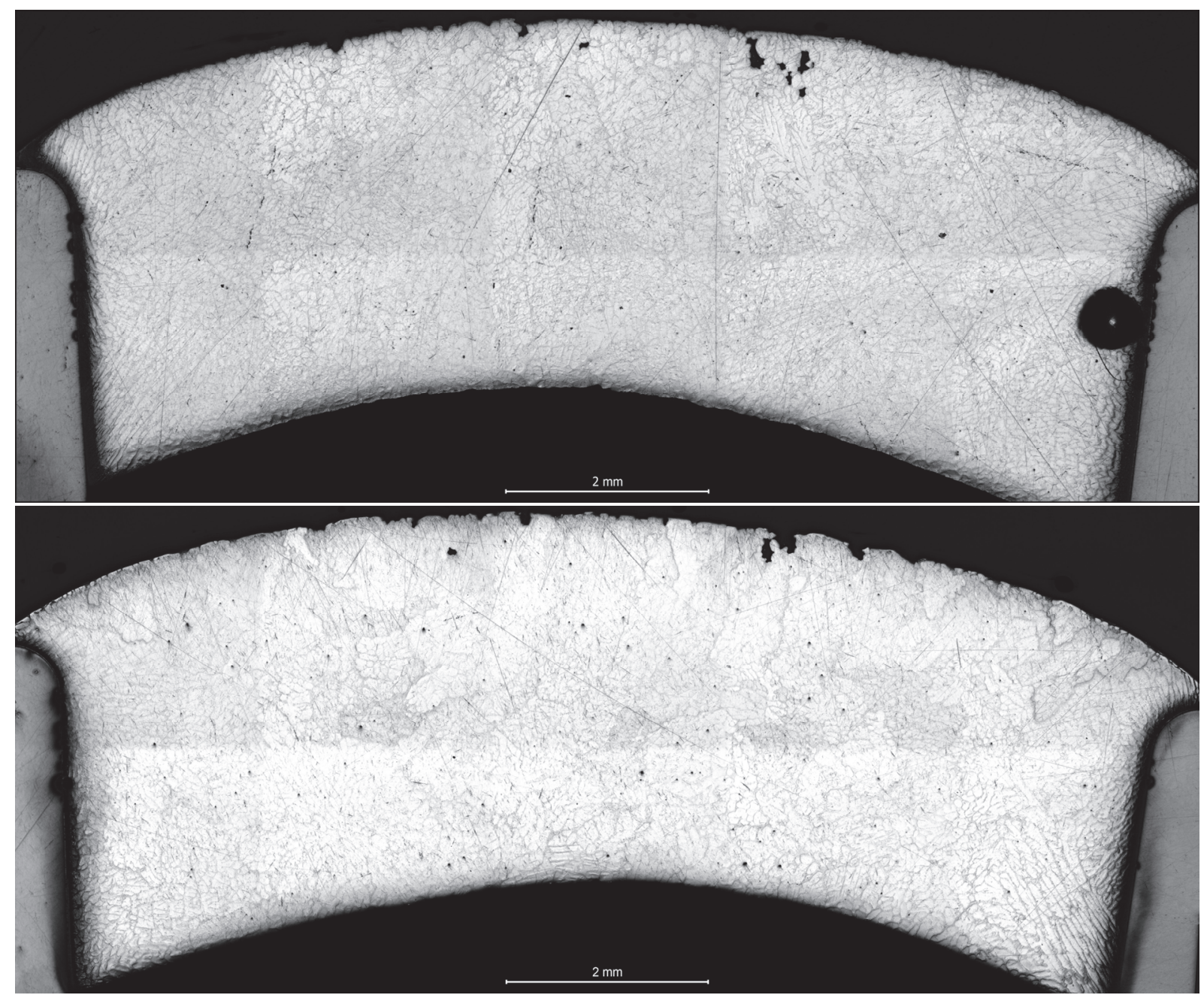

Figure S5: Both halves of the metallographic cross-section through the re-solidified Sn sample exposed to $D$ plasma for $75 \mathrm{~min}$ at $\mathbf{5 1 5} \boldsymbol{K}$. The images are stitched from several laser intensity images acquired using an Olympus LEXT OLS4000 confocal laser scanning microscope. The images show, at the bottom, part of the large gas cavity (black), and to the left and right the crucible walls (darker grey). The corrugated surface is visible, as well as large near-surface pores. These pores appear to be open to the surface, since they were filled with epoxy resin while embedding the sample for crosssectioning. Numerous smaller pores are also visible throughout the re-solidified melt. 

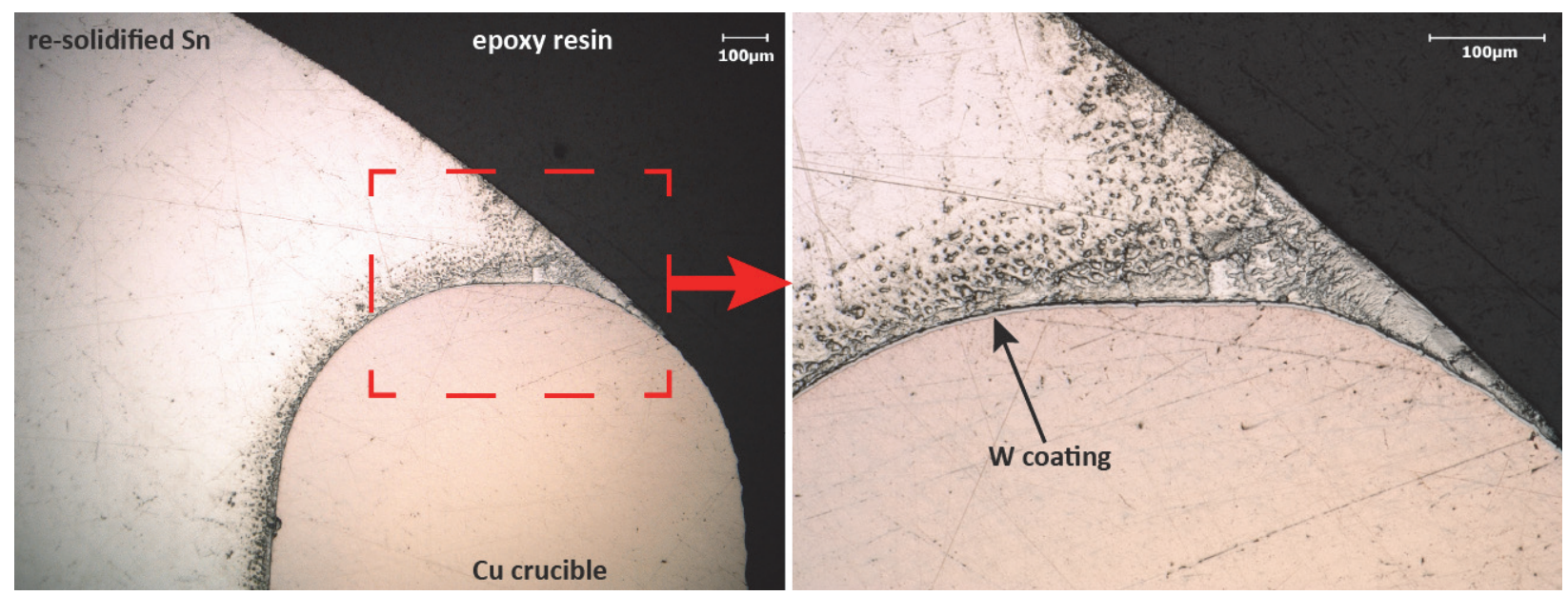

Figure S6: Close-up optical micrograph of the cross-section of Sn exposed to D plasma for $75 \mathrm{~min}$ at $515 \mathrm{~K}$. The images show the re-solidified melt at the top, the $\sim 2 \mu \mathrm{m}$-thick $\mathrm{W}$ coating and the rim of the crucible (reddish color). The dark material in the top right corner is the epoxy resin used for embedding the sample. Note the intense contact of the $\mathrm{Sn}$ to the $\mathrm{W}$, as well as the very small contact angle. The apparently porous/rough structure in the Sn close to the crucible wall is believed to be at least partly caused by etching during the polishing process due to the strongly acidic $\mathrm{pH}$ value of the polishing paste.

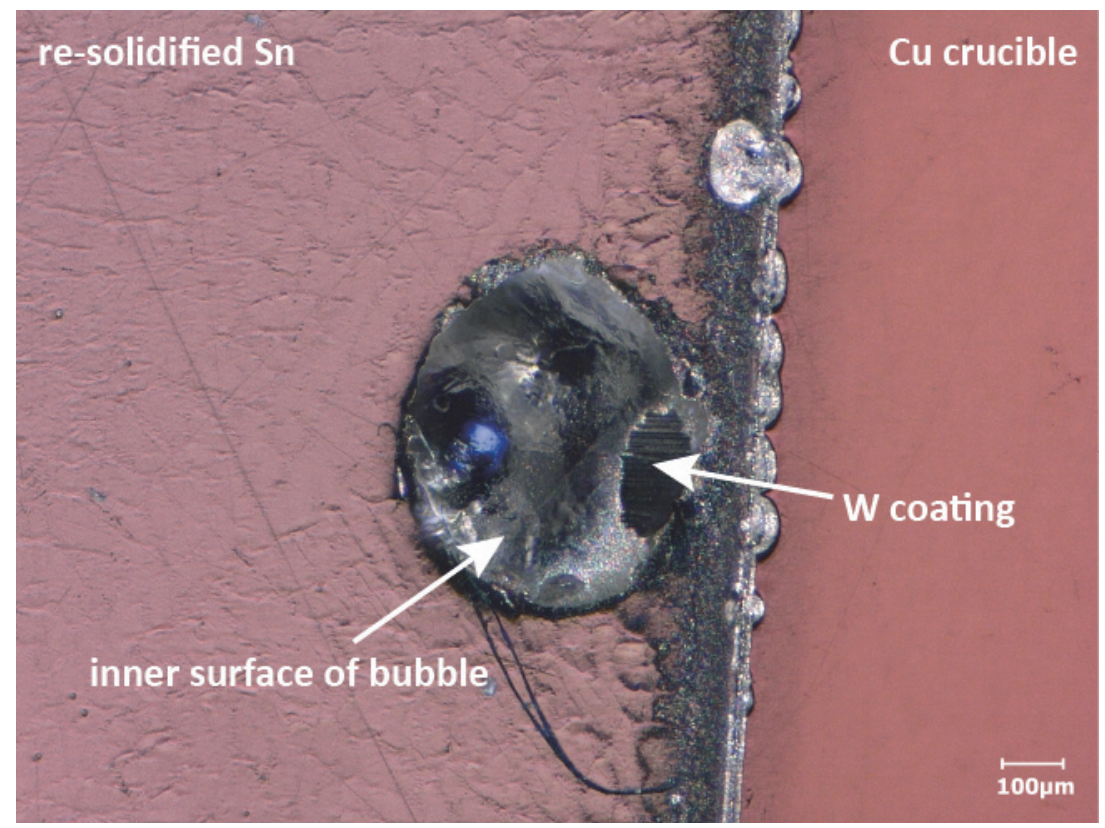

Figure S7: Close-up optical micrograph of the cross-section of Sn exposed to D plasma for $75 \mathrm{~min}$ at $\mathbf{5 1 5} \boldsymbol{K}$. The image shows the large pore visible in the top image of Figure S5. For acquiring this image, the microscope objective was tilted by $30^{\circ}$ to the left (tilt axis: vertical in the image). The image was acquired using z-stacking in order to obtain an enhanced depth of focus. It can be clearly seen that the pore is in direct contact with the W-coated crucible wall (indicated by arrow). The reddish color is caused by a polarizing filter, which suppresses overly bright direct reflections. 
Table S8: Table of time delays between sample exposure and analysis between NRA and TDS

\begin{tabular}{|c|c|c|c|c|c|c|}
\hline conditions & $\begin{array}{c}\text { plasma } \\
\text { start }\end{array}$ & $\begin{array}{l}\text { plasma } \\
\text { end }\end{array}$ & NRA & TDS & $\begin{array}{c}\text { NRA delay } \\
\text { (days) }\end{array}$ & $\begin{array}{c}\text { TDS delay } \\
\text { (days) }\end{array}$ \\
\hline $300 \mathrm{~K}, 1 \mathrm{~h}$ & 24.04 .2019 & 24.04 .2019 & 30.04 .2019 & 10.05 .2019 & 6 & 16 \\
\hline $300 \mathrm{~K}, 24 \mathrm{~h}$ & 04.04 .2019 & 05.04 .2019 & 30.04 .2019 & 13.05 .2019 & 25 & 38 \\
\hline $300 \mathrm{~K}, 96 \mathrm{~h}$ & 25.04.2019 & 29.04 .2019 & 30.04 .2019 & 14.05 .2019 & 1 & 15 \\
\hline $495 \mathrm{~K}, 1 \mathrm{~h}$ & 07.03.2019 & 07.03.2019 & 07.03.2019 & 08.05.2019 & 0 & 62 \\
\hline $\begin{array}{c}495 \mathrm{~K}, 1 \mathrm{~h} \\
\text { (repeat) }\end{array}$ & & & 03.05.2019 & & 57 & \\
\hline $\begin{array}{c}495 \mathrm{~K}, 1 \mathrm{~h} \text {, } \\
\text { empty crucible }\end{array}$ & & & & 03.05 .2019 & & 57 \\
\hline $495 \mathrm{~K}, 24 \mathrm{~h}$ & 05.03.2019 & 06.03.2019 & 07.03.2019 & 15.05 .2019 & 1 & 70 \\
\hline $\begin{array}{l}495 \mathrm{~K}, 24 \mathrm{~h} \\
\text { (repeat) }\end{array}$ & & & 03.05.2019 & & 58 & \\
\hline $495 \mathrm{~K}, 96 \mathrm{~h}$ & 15.03.2019 & 19.03.2019 & 30.04 .2019 & 16.05 .2019 & 42 & 58 \\
\hline $515 \mathrm{~K}, 1 \mathrm{~h}$ & 28.02.2019 & 28.02 .2019 & 07.03 .2019 & 06.05 .2019 & 7 & 67 \\
\hline $515 \mathrm{~K}, 1.25 \mathrm{~h}$ & 27.02.2019 & 27.02 .2019 & 07.03 .2019 & & 8 & \\
\hline
\end{tabular}

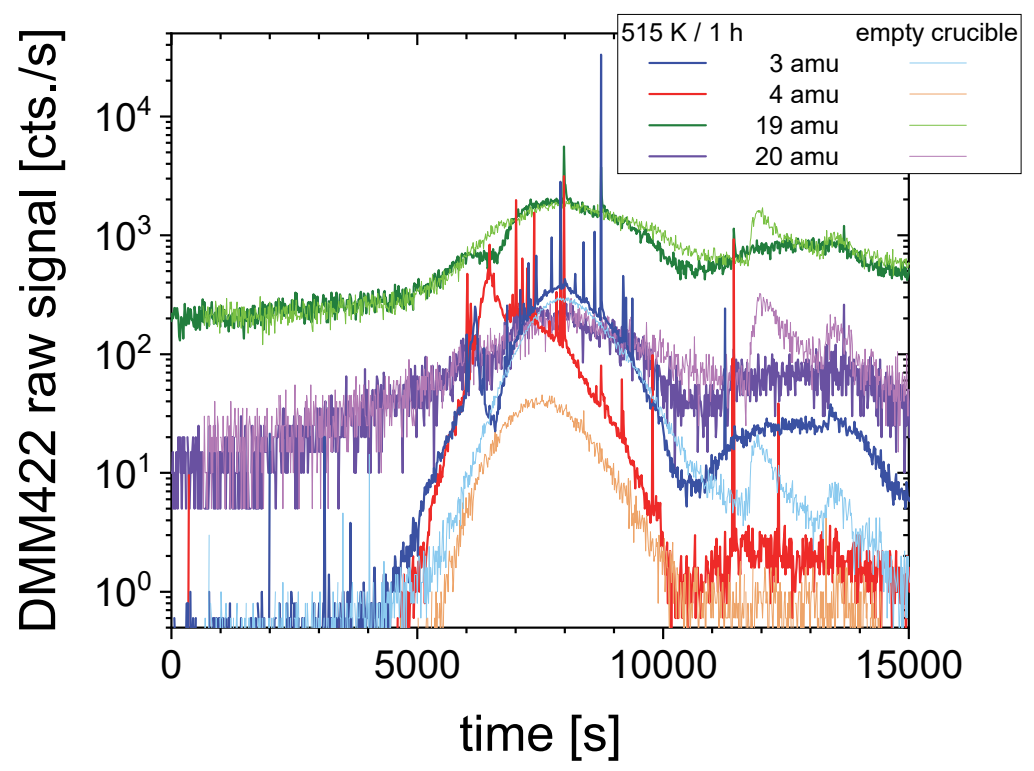

Figure 59: Release of typical D-containing molecules from Sn exposed at $515 \boldsymbol{K}$ (with crucible) and the empty crucible exposed at $495 \mathrm{~K}$, both for $1 \mathrm{~h}$. Shown are masses $3(H D), 4\left(D_{2}\right), 19(H D O)$ and 20 $\left(\mathrm{D}_{2} \mathrm{O}\right)$ amu. Note the large release of masses 3, 19 and 20 amu compared to $4 \mathrm{amu}$, which appear to originate predominantly from the crucible. Spikes of mass 3 clearly originate from the Sn sample, however. 


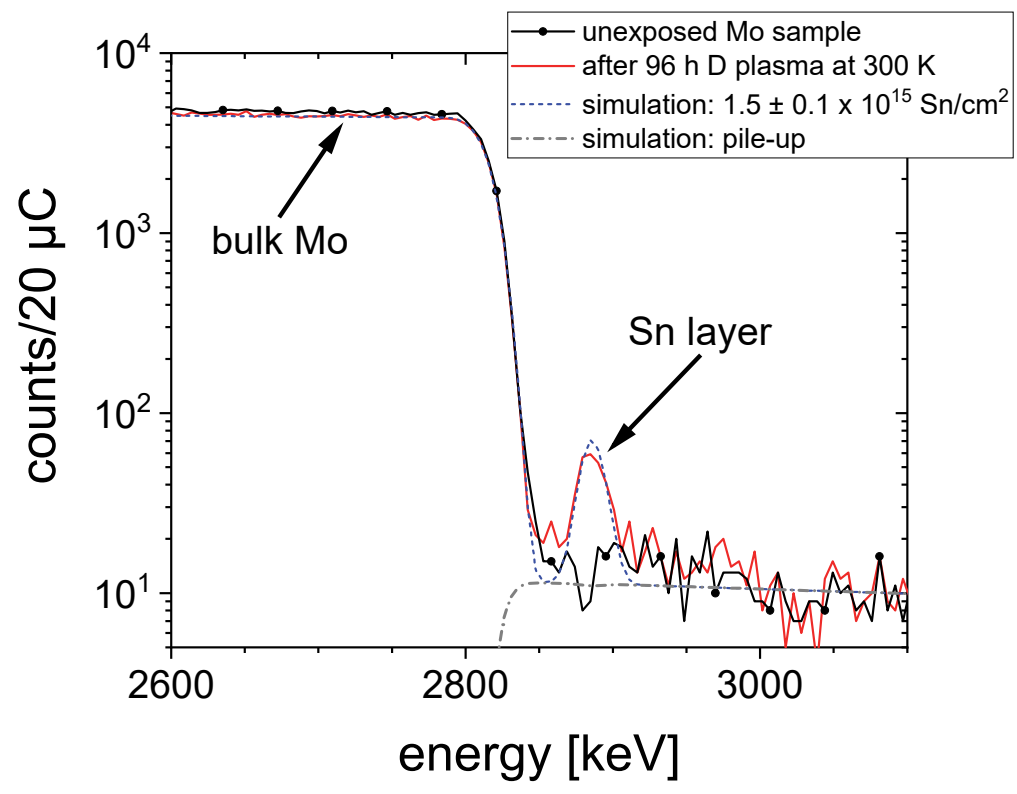

Figure S10: ${ }^{3} \mathrm{He}$ RBS spectra of Mo witness samples. All spectra were acquired at a primary beam energy of $3200 \mathrm{keV}$ with an integrated charge of $20 \mu \mathrm{C}$. The black line corresponds to an unexposed sample and only shows pile-up at energies higher than the Mo edge. The red line corresponds to the Mo sample exposed together with solid $\mathrm{Sn}$ at $300 \mathrm{~K}$ for 96 hours. Here a clear peak is visible just right of the Mo edge. The measurement is matched with good accuracy by a SIMNRA 7.0 simulation (dashed blue line) assuming a thin, pure Sn layer corresponding to 1.5 monolayers on top of the Mo. In the simulation, also the pile-up resulting from coincident detection of some of the ${ }^{3} \mathrm{He}$ particles backscattered from Mo is accurately reproduced (grey dash-dotted line).
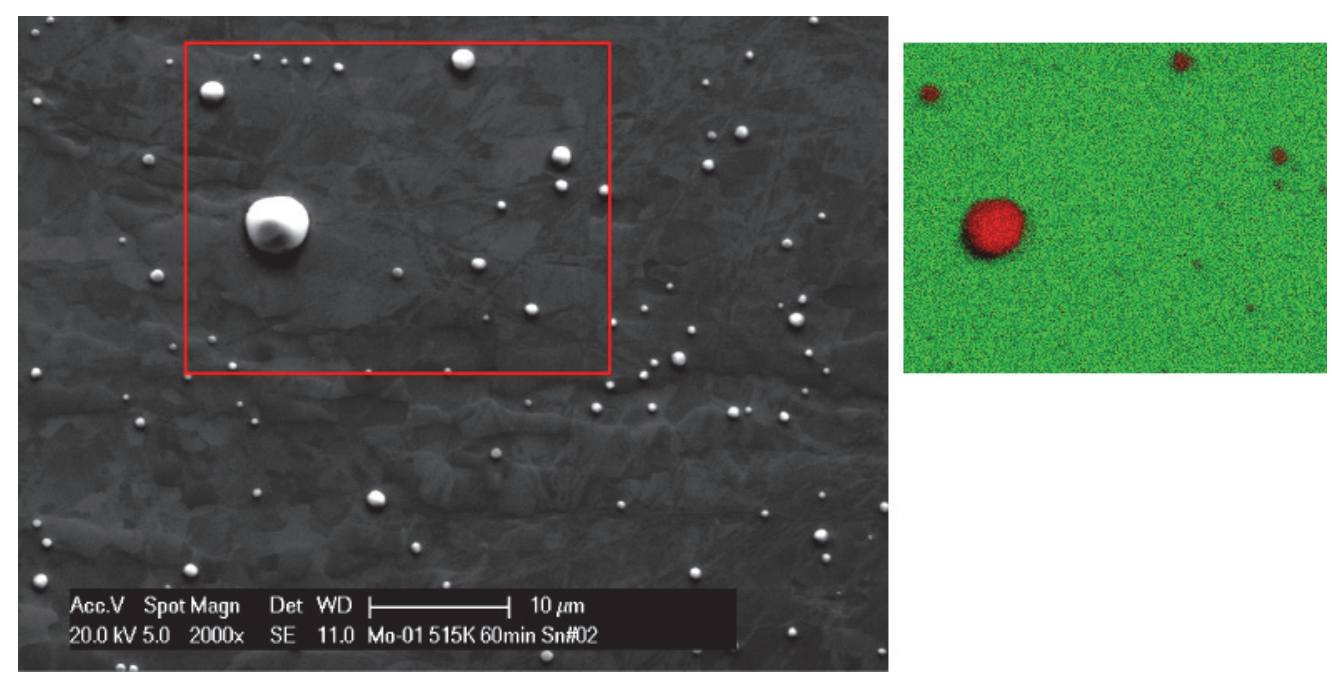

Figure S11: SEM image (left) of Sn microdroplets re-deposited onto the Mo witness sample from liquid $\mathrm{Sn}$ at $\mathbf{5 1 5} \boldsymbol{K}$ during $\mathrm{D}$ plasma exposure. The corresponding element map acquired by energydispersive X-ray analysis (EDX) is shown on the right. The Sn droplets appear bright against the darker background of the Mo sample. The red rectangle in the SEM image marks the area for EDX analysis. In the false-color element map, Mo $L_{\alpha}$ emission is represented by green, and $\mathrm{Sn}$ by red. 


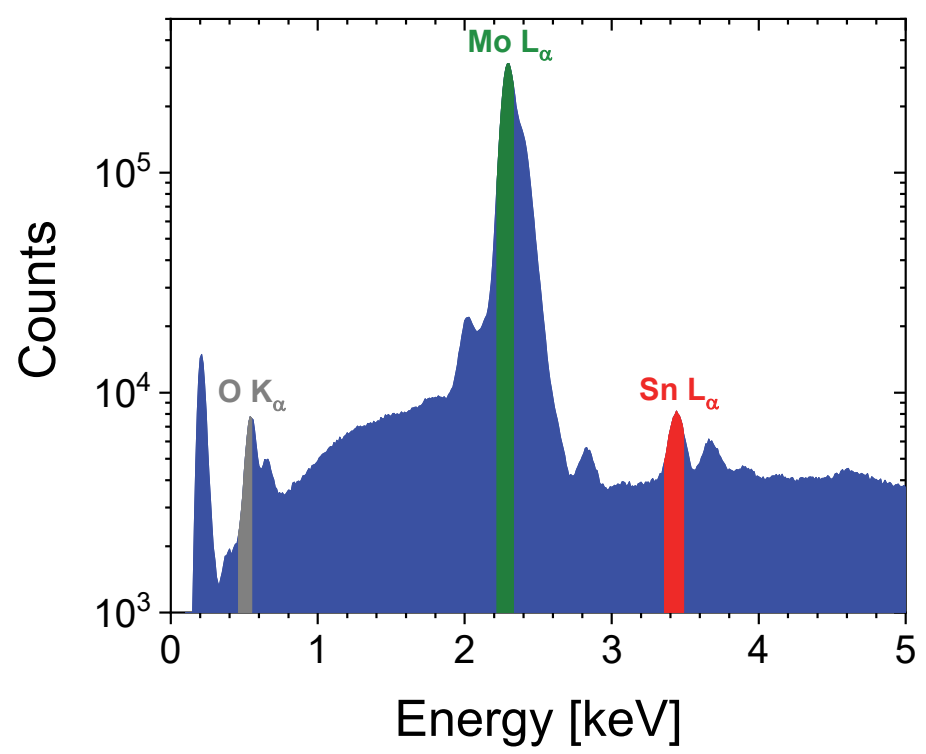

Figure S12: EDX spectrum and integration regions corresponding to the entire mapping area in Figure S9. The Mo $L_{\alpha}$ line emission is represented by green in Figure $S 11$, the $S n L_{\alpha}$ line is represented by red. X-ray emission from oxygen ( $\mathrm{OK}_{\alpha}$, grey) is not represented in Figure S11 since it is nearly uniformly distributed, but indicated for completeness in the spectrum shown here. 


\section{Index of animations and videos:}

Sn_515K_D-plasma-exposure.mp4: Time-lapse video recording of liquid tin exposed to $\mathrm{D}$ plasma at $515 \mathrm{~K}$. The recording starts close to the end of the pre-discharge, where the plasma beam is still blocked by a shutter. The plasma beam is then admitted to the sample and shortly after, the bias is switched on. This can be recognized by the plasma light becoming bright. The hemispherical surface of the Sn melt then suddenly flattens, and the Sn slowly starts rising to the rim of the crucible. At the end of the recording, the plasma is turned off and the Sn re-solidifies during cooling back to room temperature. The total duration of the plasma exposure is 1 hour.

Sn_515K_burnin-plasma_IR.mp4: Time-lapse video of liquid tin exposed to D plasma at $515 \mathrm{~K}$, recorded using the infrared camera. At 17:13 (see timestamp) the plasma shutter is opened, at 17:17 the bias of $-25 \mathrm{~V}$ is applied.

Sn_515K_meltspinning_IR_realtime: Real-time, raw video (5 frames per second, grayscale) of liquid tin exposed to $D$ plasma at $515 \mathrm{~K}$, recorded using the infrared camera. Note the small particle (indicated by the white arrow) in the center of the liquid metal surface that appears slightly brighter than its surroundings. It spins approximately once per second.

Sn_495K_D-plasma-exposure_part1.mp4: Time-lapse video recording of solid tin exposed to D plasma at $495 \mathrm{~K}$. The recording starts just before opening the beam shutter and admitting the plasma directly to the sample. Opening of the shutter can be recognized by the brightness increase due to the plasma light. The originally mirror-like, shiny Sn surface, which also shows some large grains that were formed after the pre-melting, gradually turns dull due to the formation of a highly porous, sponge-like layer at the surface. The recording shows the first $\sim 1.5$ hours of a $96 \mathrm{~h}$ plasma exposure.

Sn_495K_D-plasma-exposure_part2.mp4: Time-lapse video recording of solid tin exposed to D plasma at $495 \mathrm{~K}$. The recording continues after the end of the previous video, i.e., $\sim 1.5$ hours after the start of the plasma exposure. It shows the next 94 hours of the $96 \mathrm{~h}$ exposure with a larger time interval between frames. During this time, the surface becomes even darker and more dull due to evolution of the sponge-like layer. The Sn sample also appears to swell slightly (compare, e.g., frames at the beginning and at the end of the video).

Sn_495K_1h_slicing.mp4: Slice-and-view of the porous layer on the Sn sample exposed to D plasma at $495 \mathrm{~K}$ for $1 \mathrm{~h}$. The video was created from an SEM image sequence acquired during progressively cross-sectioning the sample using the FIB. The viewing angle is $40^{\circ}$ relative to the surface normal. The angle between electron and ion beam is $52^{\circ}$, so the cross-section is inclined by $12^{\circ}$ relative to the surface normal. For imaging, the sample was tilted by $40^{\circ}$ relative to the surface normal, causing the cross-section to appear under a viewing angle of $-38^{\circ}$. Prior to slicing, the sample was covered with a protective amorphous $\mathrm{Pt}$ :C layer, which appears slightly darker than the $\mathrm{Sn}$. It can be seen that some of the pores are connected to the surface, while others are completely surrounded by Sn.

Sn_495K_24h_slicing.mp4: Slice-and-view of the further-developed sponge-like layer on the Sn sample exposed to D plasma at $495 \mathrm{~K}$ for $24 \mathrm{~h}$. This sample was also covered by Pt:C prior to FIB cutting. The viewing angle is $40^{\circ}$ relative to the surface normal. The angle between electron and ion beam is $52^{\circ}$, so the cross-section is inclined by $12^{\circ}$ relative to the surface normal, and accordingly viewed under an angle of $-38^{\circ}$. The sponge-like layer is much thicker than after $1 \mathrm{~h}$ of plasma exposure and shows a more complex pore structure.

Sn_495K_96h_tilting.mp4: Tilt series of SEM images of a FIB cut into the Sn sample exposed to D plasma at $495 \mathrm{~K}$ for $96 \mathrm{~h}$. The cut was prepared with an inclination of $12^{\circ}$ to the surface normal, i.e., 
at $0^{\circ}$ tilt angle, the cross section appears under a viewing angle of $12^{\circ}$. This sample was not covered by a protective Pt:C layer prior to FIB cutting. Only a few pores are still clearly visible due to extensive re-deposition of Sn during cutting. The final cut is up to approximately $500 \mu \mathrm{m}$ deep, but the exact depth is difficult to measure due to the extreme corrugation of the surface and due to the steep viewing angle necessary for seeing the entire cross-section. The cross-section preparation by FIB proved to be extremely challenging, both due to the extreme depth of the cut, as well as due to strong charging effects. These caused the ion beam position to become unstable, which primarily manifested in a displacement of the cut by about $50 \mu \mathrm{m}$ shortly after the FIB milling began. Afterwards, the position remained approximately stable for longer periods of time, interrupted by occasional smaller jumps as well as brightness flashes.

Sn_300K_ini-exposed.mp4: Slide show of in-situ images of the Sn samples exposed at $300 \mathrm{~K}$. The first two images correspond to the sample exposed for 1 hour before and after plasma exposure. The following two images correspond to the sample exposed for 96 hours before and after plasma exposure. The brighter appearance of the $\mathrm{Sn}$ after plasma exposure is caused by roughening of the surface, which leads to increased diffuse reflection of light. The initial, shiny surfaces exhibit practically only specular reflection and therefore appear dark apart from the small parts that are in direct reflection condition.

Sn_300K_96h_topview.mp4: Sequential SEM images acquired on the surface of the Sn sample exposed to D plasma at $300 \mathrm{~K}$ for $96 \mathrm{~h}$. Note the small pores indicated by arrows. From one SEM frame to the next, they increase in size and/or contrast. This instability made it very challenging to accurately image details of the sample surface, and also highlights the fragility of these pores.

Sn_300K_96h_XS.mp4: Sequential SEM images acquired on a FIB cross-section cut into the Sn sample exposed to $\mathrm{D}$ plasma at $300 \mathrm{~K}$ for $96 \mathrm{~h}$. The cross-section appears under a viewing angle of $-38^{\circ}$. The images were recorded immediately after the cross-section preparation was finished. The first image corresponds to Figure $3 \mathrm{~b}$ in the main manuscript. In the following images, more and more of the initially visible pores disappear, and also the rest of the cross-section surface changes its appearance. 